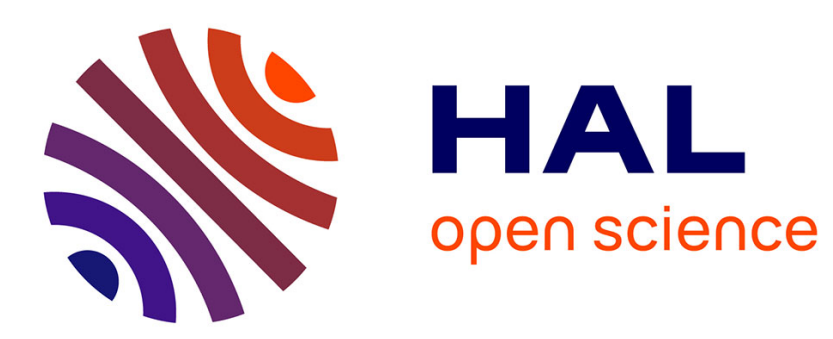

\title{
Bubble collapse near a solid boundary: a numerical study of the influence of viscosity
}

S. Popinet, S. Zaleski

\section{To cite this version:}

S. Popinet, S. Zaleski. Bubble collapse near a solid boundary: a numerical study of the influence of viscosity. Journal of Fluid Mechanics, 2002, 464, pp.137-163. 10.1017/S002211200200856X . hal01445438

\author{
HAL Id: hal-01445438 \\ https://hal.science/hal-01445438
}

Submitted on 7 Feb 2017

HAL is a multi-disciplinary open access archive for the deposit and dissemination of scientific research documents, whether they are published or not. The documents may come from teaching and research institutions in France or abroad, or from public or private research centers.
L'archive ouverte pluridisciplinaire HAL, est destinée au dépôt et à la diffusion de documents scientifiques de niveau recherche, publiés ou non, émanant des établissements d'enseignement et de recherche français ou étrangers, des laboratoires publics ou privés. 


\section{Bubble collapse near a solid boundary: A numerical study of the influence of viscosity}

\section{By STÉPHANE POPINET AND STÉPHANE ZALESKI}

Laboratoire de Modélisation en Mécanique,

CNRS and Université Pierre et Marie Curie (Paris VI)

8 rue du Capitaine Scott, 75015 Paris, France,

(popinet | zaleski)@lmm.jussieu.fr

(Received 8 January 2001)

The effect of viscosity on jet formation for bubbles collapsing near solid boundaries is studied numerically. A numerical technique is presented which allows the Navier-Stokes equations with free-surface boundary conditions to be solved accurately and efficiently. Good agreement is obtained between experimental data and numerical simulations for the collapse of large bubbles. However, it is shown that compressible and thermal effects must be taken into account in order to describe the energy dissipation occurring during jet impact correctly. A parametric study of the effect of viscosity on jet impact velocity is undertaken. The jet impact velocity is found to decrease as viscosity increases and above a certain threshold jet impact is impossible. We study how this critical Reynolds number depends on the initial radius and the initial distance from the wall. A simple scaling law is found to link this critical Reynolds number to the other non-dimensional parameters of the problem.

\section{Introduction}

Violent collapse of bubbles in asymmetrical geometries occur in a number of situations of practical interest including cavitation, shock-wave and laser lithotripsy. When close enough to a solid boundary these collapses are usually associated with high-speed jet formation. While jet formation for cavitation bubbles was demonstrated experimentally as early as 1961 (Naudé \& Ellis 1961) there is still debate regarding the importance of jet impact as the main mechanism for cavitation damage. The first explanation was given by Rayleigh (1917) who considered the high pressure caused by the collapse to be the main damaging mechanism. An alternative explanation was given by Kornfeld \& Suvorov (1944) who suggested the jet formation effect which was experimentally demonstrated by Naudé \& Ellis. Benjamin \& Ellis (1966) then concluded that jet formation and impact was important and probably the main factor for cavitation damage. Recently, however, a number of experiments have cast doubt on this explanation. In these investigations, the damages were found to be distributed around a circumference and not on the axis of symmetry as would be the case if jet impact was the main factor for cavitation damage (Tomita \& Shima 1986). Philipp \& Lauterborn conclude that the main mechanism for cavitation damage are the high-pressures and temperatures reached inside a bubble collapsing very close to the solid boundary (Philipp \& Lauterborn 1998).

A number of points regarding bubble collapse in bounded domains thus remain open questions. However, the analytical study of the problem of bubble collapse in the vicinity 
of a solid boundary is very difficult. Consequently, apart from the perturbation approach of Rattray (1951), most of the results were obtained using numerical solutions of boundary integral formulations (Plesset \& Chapman 1971; Blake et al. 1993). These methods rest on the surface integration of a potential solution for the fluid flow and can be used only for vanishing viscosities or vanishing advection terms (Stokes flow). In all the studies so far the effect of viscosity has thus been neglected. While good agreement was found between numerical and experimental results, due to obvious practical considerations experiments are usually performed with large bubbles (millimeter sized). In these cases, viscosity is unlikely to play a major role and inviscid calculations give good results. However, one can ask how these results would scale for smaller bubbles where viscosity is likely to come into play (cavitating bubbles are likely to be much smaller than one millimeter). Experiments with very small bubbles are more difficult to perform and analytical approaches including viscous effects are even more intricate, but we believe that the use of an appropriate numerical technique can give useful insights.

In this article, we describe an original numerical technique which allows the resolution of the incompressible Navier-Stokes equations in axisymmetric coordinates with freesurface boundary conditions. We then present the results of first comparisons between our numerical results and experiments for bubble collapse and jet formation near a wall. Then, because the code is fast enough to allow a parametric study of the influence of viscosity on jet formation and evolution, we present a phase diagram function of the independent non-dimensional parameters illustrating the effect of viscosity on the impact velocity.

\section{Numerical technique}

We present a numerical method for solving the axisymmetric Navier-Stokes equations with free-surface boundary conditions. While this has been done in the past, the methods used either made crude assumptions about free-surface boundary conditions (Harlow \& Welch 1965; Chan \& Street 1970; Nichols \& Hirt 1971; Hirt \& Nichols 1981) or used boundary fitted grids (Blanco \& Magnaudet 1995; Legendre 1996). Our method is based on a finite volume formulation using both a fixed grid and a front-tracking approach. The free-surface is tracked using surface points (markers) connected with cubic splines. This allow us to deal with surface integral terms appearing in the finite volume formulation correctly. Moreover, this method is not limited to simple geometries and can deal efficiently with large deformations of the interface. This technique constitutes an extension of the two-fluid approach of Popinet \& Zaleski (1999).

\subsection{The explicit equations}

The incompressible Navier-Stokes equations for an axisymmetric flow in cylindrical coordinates can be written as

$$
\begin{aligned}
\frac{1}{r} \frac{\partial\left(r v_{r}\right)}{\partial r}+\frac{\partial v_{z}}{\partial z} & =0 \\
\frac{\partial v_{r}}{\partial t}+\frac{1}{r} \frac{\partial\left(r v_{r}^{2}\right)}{\partial r}+\frac{\partial\left(v_{r} v_{z}\right)}{\partial z} & =-\frac{\partial \Phi}{\partial r}+\frac{1}{r} \frac{\partial\left(r S_{r r}\right)}{\partial r}+\frac{\partial S_{z r}}{\partial z}-\frac{S_{\theta \theta}}{r} \\
\frac{\partial v_{z}}{\partial t}+\frac{1}{r} \frac{\partial\left(r v_{r} v_{z}\right)}{\partial r}+\frac{\partial\left(v_{z}^{2}\right)}{\partial z} & =-\frac{\partial \Phi}{\partial z}+\frac{1}{r} \frac{\partial\left(r S_{z r}\right)}{\partial r}+\frac{\partial S_{z z}}{\partial z}
\end{aligned}
$$

where $\Phi=p / \rho, \rho$ is constant and the components of the stress tensor are defined as

$$
S_{r r}=2 \nu \frac{\partial v_{r}}{\partial r}
$$




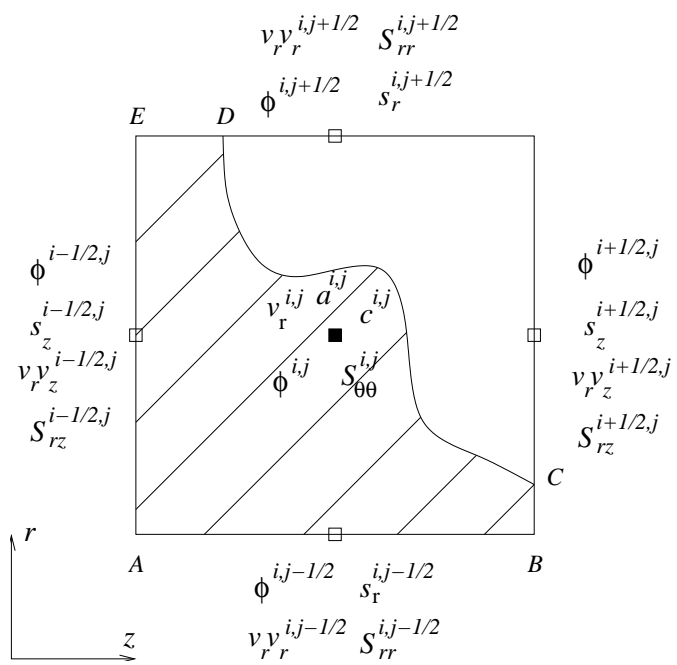

FIGURE 1. Finite volume discretisation.

$$
\begin{aligned}
& S_{\theta \theta}=2 \nu \frac{v_{r}}{r} \\
& S_{z z}=2 \nu \frac{\partial v_{z}}{\partial z} \\
& S_{z r}=S_{r z}=\nu\left(\frac{\partial v_{z}}{\partial r}+\frac{\partial v_{r}}{\partial z}\right),
\end{aligned}
$$

where $\nu$ is the kinematic viscosity.

\subsection{Finite volume formulation}

In order to obtain a finite volume formulation necessary for numerical analysis, we first need to integrate the equations over an arbitrarily moving domain. Let us call the domain $\Omega$ and $\partial \Omega$ its boundary. $\mathbf{u}$ is the velocity of the boundary $\partial \Omega$. Integrating over $\Omega$ yields the integral equations

$$
\begin{array}{r}
\frac{\partial}{\partial t} \int_{\Omega} r d r d z+\int_{\partial \Omega} r\left(v_{z}-u_{z}\right) d r-\int_{\partial \Omega} r\left(v_{r}-u_{r}\right) d z=0 \\
\frac{\partial}{\partial t} \int_{\Omega} v_{r} r d r d z+\int_{\partial \Omega} r v_{r}\left(v_{z}-u_{z}\right) d r-\int_{\partial \Omega} r v_{r}\left(v_{r}-u_{r}\right) d z= \\
\int_{\partial \Omega} \Phi r d z+\int_{\Omega} \Phi d r d z+\int_{\partial \Omega} r S_{z r} d r-\int_{\partial \Omega} r S_{r r} d z-\int_{\Omega} S_{\theta \theta} d r d z \\
\frac{\partial}{\partial t} \int_{\Omega} v_{z} r d r d z+\int_{\partial \Omega} r v_{z}\left(v_{z}-u_{z}\right) d r-\int_{\partial \Omega} r v_{z}\left(v_{r}-u_{r}\right) d z= \\
-\int_{\partial \Omega} \Phi r d r+\int_{\partial \Omega} r S_{z z} d r-\int_{\partial \Omega} r S_{z r} d z
\end{array}
$$

Then, considering a square finite volume centered at $\left(z_{i}, r_{j}\right)$ of side $h$, we seek the integral formulation above for the $v_{r}$ component of the velocity. In the general case of a free-surface flow problem this control volume can be cut by the interface. In this case the volume of integration is a piece of the square $\Omega$ with boundary $A B C D E A$ (Figure 1). The velocity $\mathbf{u}$ of the boundary is 0 on $A B \cup B C \cup D E \cup E A$ and $\mathbf{v}$ on $C D$. Equation 
(2.9) can then be written

$$
\begin{gathered}
\frac{\partial}{\partial t} \int_{\Omega} v_{r} r d r d z+\int_{B C \cup E A} r v_{r} v_{z} d r-\int_{A B \cup D E} r v_{r}^{2} d z= \\
\int_{A B \cup D E} \Phi r d z+\int_{C D} \Phi r d z+\int_{\Omega} \Phi d r d z+ \\
\int_{B C \cup E A} r S_{z r} d r+\int_{C D} r S_{z r} d r- \\
\int_{A B \cup D E} r S_{r r} d z-\int_{C D} r S_{r r} d z-\int_{\Omega} S_{\theta \theta} d r d z .
\end{gathered}
$$

We then assume that the different quantities needed are defined either at the center $\left(z_{i}, r_{j}\right)$ of the cell or at the center of the cell faces $\left(z_{i}, r_{j} \pm h / 2\right)$ and $\left(z_{i} \pm h / 2, r_{j}\right)$, in a typical staggered grid fashion (Peyret \& Taylor 1983). We also make the assumption that quantities defined at the center of the cell are constant over the whole cell whereas the quantities defined on the cell faces are constant on these faces. Introducing the cell faces area $s_{r}^{i, j}, s_{z}^{i, j}$, cell area $a^{i, j}$ and cell volume $c^{i, j}$ defined as the area of the cell faces, area or volume of the cell occupied by the fluid, in the general case we obtain

$$
\begin{aligned}
& \frac{\partial}{\partial t}\left[\left(c v_{r}\right)^{i, j}\right]+\left(s_{z} v_{r} v_{z}\right)^{i+1 / 2, j}-\left(s_{z} v_{r} v_{z}\right)^{i-1 / 2, j}+ \\
& \left(s_{r} v_{r}^{2}\right)^{i, j+1 / 2}-\left(s_{r} v_{r}^{2}\right)^{i, j-1 / 2}= \\
& \left(s_{r} \Phi\right)^{i, j-1 / 2}-\left(s_{r} \Phi\right)^{i, j+1 / 2}+(a \Phi)^{i, j}+\int_{i n t} \Phi r d z+ \\
& \left(s_{z} S_{z r}\right)^{i+1 / 2, j}-\left(s_{z} S_{z r}\right)^{i-1 / 2, j}+\int_{i n t} r S_{z r} d r+ \\
& \left(s_{r} S_{r r}\right)^{i, j+1 / 2}-\left(s_{r} S_{r r}\right)^{i, j-1 / 2}-\int_{i n t} r S_{r r} d z-\left(a S_{\theta \theta}\right)^{i, j}
\end{aligned}
$$

where $\int_{\text {int }}$ denotes the integration along the piece of interface contained in $\Omega$. The equation for the $z$ component of the velocity, obtained in a very similar manner is

$$
\begin{aligned}
& \frac{\partial}{\partial t}\left[\left(c v_{z}\right)^{i, j}\right]+\left(s_{z} v_{z}^{2}\right)^{i+1 / 2, j}-\left(s_{z} v_{z}^{2}\right)^{i-1 / 2, j}+ \\
& \left(s_{r} v_{r} v_{z}\right)^{i, j+1 / 2}-\left(s_{r} v_{r} v_{z}\right)^{i, j-1 / 2}= \\
& \left(s_{z} \Phi\right)^{i-1 / 2, j}-\left(s_{z} \Phi\right)^{i+1 / 2, j}-\int_{i n t} \Phi r d r+ \\
& \left(s_{z} S_{z z}\right)^{i+1 / 2, j}-\left(s_{z} S_{z z}\right)^{i-1 / 2, j}+\int_{i n t} r S_{z z} d r+ \\
& \left(s_{r} S_{z r}\right)^{i, j+1 / 2}-\left(s_{r} S_{z r}\right)^{i, j-1 / 2}-\int_{i n t} r S_{z r} d z
\end{aligned}
$$

Noting that

$$
\frac{\partial}{\partial t} \int_{\Omega} r d r d z=\int_{\partial \Omega} r u_{z} d r-\int_{\partial \Omega} r u_{r} d z
$$

(2.8) can be rewritten as

$$
\int_{\partial \Omega} r v_{z} d r-\int_{\partial \Omega} r v_{r} d z=0
$$


which yields the discrete volume incompressibility condition

$$
\begin{gathered}
\left(s_{z} v_{z}\right)^{i+1 / 2, j}-\left(s_{z} v_{z}\right)^{i-1 / 2, j}+\int_{i n t} r v_{z} d r+ \\
\left(s_{r} v_{r}\right)^{i, j+1 / 2}-\left(s_{r} v_{r}\right)^{i, j-1 / 2}-\int_{i n t} r v_{r} d z=0 .
\end{gathered}
$$

\subsection{The Discrete Pressure Equation}

We use a projection method to solve the incompressibility condition. The Poisson-like equation for the pressure can be expressed as a function of the numerical divergence of the temporary velocity field $\mathbf{v}^{\star}$. The velocity field at time $n+1$ is obtained from the relations

$$
\begin{aligned}
v_{r}^{i, j} & =\left(v_{r}^{\star}\right)^{i, j}+\frac{\Delta t}{c^{i, j}}\left[\left(s_{r} \Phi\right)^{i, j-1 / 2}-\left(s_{r} \Phi\right)^{i, j+1 / 2}+(a \Phi)^{i, j}\right] . \\
v_{z}^{i, j} & =\left(v_{z}^{\star}\right)^{i, j}+\frac{\Delta t}{c^{i, j}}\left[\left(s_{z} \Phi\right)^{i-1 / 2, j}-\left(s_{z} \Phi\right)^{i+1 / 2, j}\right] .
\end{aligned}
$$

We make the assumption that the two components of the velocity vary linearly from one side of the cell to the other, yielding the expressions for the velocity in a $\left(z_{i}, r_{j}\right)$ cell

$$
\begin{aligned}
& v_{z}(z) h=\left(z-z_{i-1 / 2}\right) v_{z}^{i+1 / 2, j}-\left(z-z_{i+1 / 2}\right) v_{z}^{i-1 / 2, j}, \\
& v_{r}(r) h=\left(r-r_{j-1 / 2}\right) v_{r}^{i, j+1 / 2}-\left(r-r_{j+1 / 2}\right) v_{r}^{i, j-1 / 2} .
\end{aligned}
$$

The integrals along the section of interface in the $\left(z_{i}, r_{j}\right)$ cell can be computed as

$$
\begin{aligned}
& \int_{\text {int }_{i, j}} r v_{z} d r= \\
& \frac{v_{z}^{i+1 / 2, j}}{h} \int_{\text {int }_{i, j}} r\left(z-z_{i-1 / 2}\right) d r-\frac{v_{z}^{i-1 / 2, j}}{h} \int_{\text {int }_{i, j}} r\left(z-z_{i+1 / 2}\right) d r, \\
& \int_{\text {int }_{i, j}} r v_{r} d z= \\
& \frac{v_{r}^{i, j+1 / 2}}{h} \int_{\text {int }_{i, j}} r\left(r-r_{j-1 / 2}\right) d z-\frac{v_{r}^{i, j-1 / 2}}{h} \int_{\text {int }_{i, j}} r\left(r-r_{j+1 / 2}\right) d z .
\end{aligned}
$$

If we assume that $\Phi^{i, j+1 / 2}=\left(\Phi^{i, j}+\Phi^{i, j+1}\right) / 2$, the incompressibility condition (2.16) can be written as

$$
\begin{aligned}
& \frac{1}{\Delta t}\left[\left(s_{z}^{\prime} v_{z}^{\star}\right)^{i+1 / 2, j}-\left(s_{z}^{\prime} v_{z}^{\star}\right)^{i-1 / 2, j}+\left(s_{r}^{\prime} v_{r}^{\star}\right)^{i, j+1 / 2}-\left(s_{r}^{\prime} v_{r}^{\star}\right)^{i, j-1 / 2}\right]+ \\
& \Phi^{i, j}\left[s_{z}^{i, j}\left(\left(s_{z}^{\prime} / c\right)^{i+1 / 2, j}+\left(s_{z}^{\prime} / c\right)^{i-1 / 2, j}\right)+s_{r}^{i, j}\left(\left(s_{r}^{\prime} / c\right)^{i, j+1 / 2}+\left(s_{r}^{\prime} / c\right)^{i, j-1 / 2}\right)\right]+ \\
& \frac{\Phi^{i, j}}{2}\left[\left(a s_{r}^{\prime} / c\right)^{i, j+1 / 2}-\left(a s_{r}^{\prime} / c\right)^{i, j-1 / 2}\right]= \\
& \left(s_{z} \Phi\right)^{i+1, j}\left(s_{z}^{\prime} / c\right)^{i+1 / 2, j}+ \\
& \left(s_{z} \Phi\right)^{i-1, j}\left(s_{z}^{\prime} / c\right)^{i-1 / 2, j}+\Phi^{i, j+1}\left(s_{r}^{\prime} / c\right)^{i, j+1 / 2}\left(s_{r}^{i, j+1}-\frac{1}{2} a^{i, j+1 / 2}\right)+ \\
& \Phi^{i, j-1}\left(s_{r}^{\prime} / c\right)^{i, j-1 / 2}\left(s_{r}^{i, j-1}+\frac{1}{2} a^{i, j-1 / 2}\right),
\end{aligned}
$$




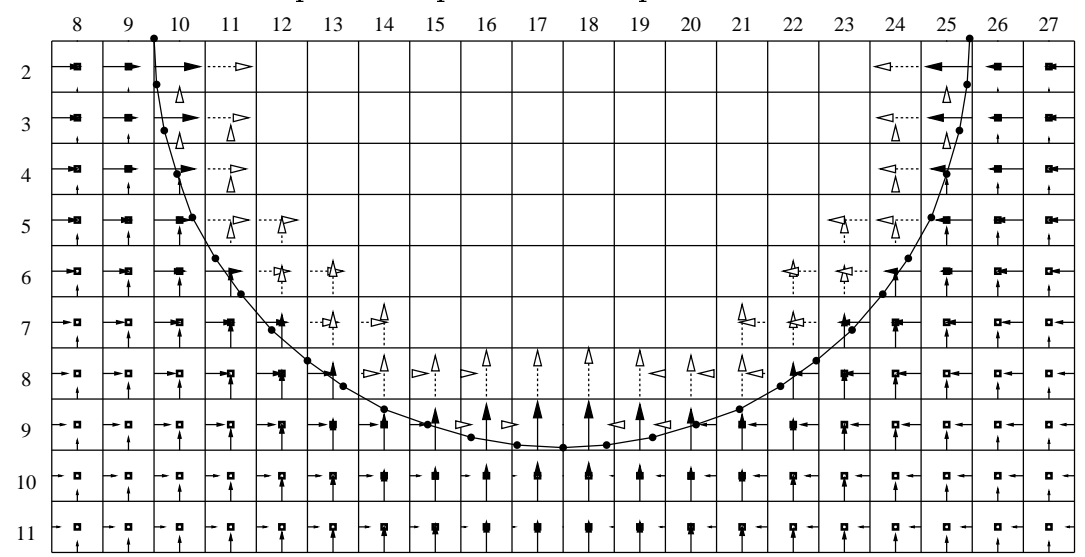

FIGURE 2. Mesh and discretisation of the velocity and pressure fields. The marker points and the connecting cubic splines are represented. The light arrows are extrapolated values of the velocity field. The squares indicate the location of the pressure nodes where the pressure equation is solved.

where $s_{z}^{\prime}$ and $s_{r}^{\prime}$ are defined as

$$
\begin{aligned}
& \left(s_{z}^{\prime}\right)^{i \pm 1 / 2, j}=s_{z}^{i \pm 1 / 2, j}+\frac{1}{h} \int_{\text {int }_{i, j}} r\left(z-z_{i \mp 1 / 2}\right) d r \\
& \left(s_{r}^{\prime}\right)^{i, j \pm 1 / 2}=s_{r}^{i, j \pm 1 / 2}-\frac{1}{h} \int_{\text {int }_{i, j}} r\left(r-r_{j \mp 1 / 2}\right) d z .
\end{aligned}
$$

\subsection{Numerical method of resolution}

The Poisson-like pressure equation (2.21) is solved using multigrid-accelerated GaussSeidel relaxation (Press \& Teukolsky sep/oct 1991).

The interface is discretised using a set of marker points linked by cubic splines. This description allows the precise knowledge of the position and curvature of the interface required in order to include the surface tension terms and all the other surface integral terms appearing in (2.12), (2.13) and (2.21).

As in all free-surface codes (Harlow \& Welch 1965; Chan \& Street 1970; Nichols \& Hirt 1971; Hirt \& Nichols 1981), the most delicate point is the treatment of free-surface boundary conditions. The pressure on the interface on the fluid side is given by

$$
p=p_{i}+\sigma \kappa+\mu \mathbf{n . D . n},
$$

where $p_{i}$ is the pressure in the bubble, $\sigma$ the surface tension coefficient, $\kappa$ the curvature, $\mu$ the dynamic viscosity, $\mathbf{n}$ the normal to the interface and $\mathbf{D}$ the deviatory part of the stress tensor. This boundary condition is used directly when calculating the surface integral pressure contribution to (2.12) and (2.13).

Since we use a fixed grid to solve the Navier-Stokes equations, we need to extrapolate the velocity field far enough inside the bubble to get the velocity values necessary for the marker points advection and for the resolution of the Navier-Stokes equations on the fluid boundary (Figure 2). Moreover, this must be done while fulfilling the zero tangential stress interface boundary condition

$$
\text { t.D.n }=0 \text {, }
$$

where $\mathbf{t}$ is the tangent to the interface. Given a point $P$ near the interface, we assume that locally around $P$ the velocity field can be described as $\mathbf{u}=\mathbf{u}_{0}+\mathbf{A} \cdot \mathbf{x}$ where $\mathbf{x}$ is 


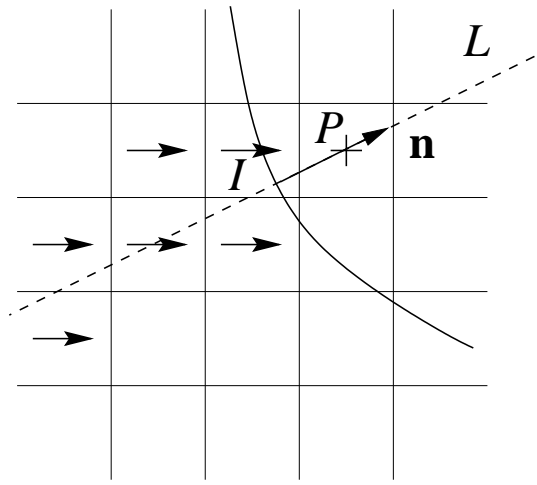

Figure 3. Selection of points for the extrapolation technique around point $P$.

the position vector and $\mathbf{A}$ is a $2 \times 2$ matrix. For this particular velocity field equation (2.25) can be expressed as $e_{i j} t_{i} n_{j}=0$ with

$$
e_{i j}=\frac{\partial u_{i}}{\partial x_{j}}+\frac{\partial u_{j}}{\partial x_{i}}=A_{i j}+A_{j i}
$$

Given a set of $N$ points in the vicinity of $P$ and a vector $t$ tangent to the interface, $\mathbf{u}_{0}$ and $\mathbf{A}$ can be found by minimisation of the function

$$
\mathcal{L}=\sum_{n=1}^{N}\left(\mathbf{u}_{0}+\mathbf{A} \cdot \mathbf{x}_{n}-\mathbf{u}_{n}\right)^{2}+\lambda e_{i j} t_{i} n_{j},
$$

where $\lambda$ is a Lagrange multiplier. The set of points is chosen as on Figure 3. Line $L$ has direction $\mathbf{n}(I)$ and goes through points $P$ and $I$. A small number of points (typically five) is chosen around $L$ by minimisation of the cost function

$$
\sum d\left(P_{i}, L\right)^{2}+\zeta d\left(P_{i}, P\right)^{2}
$$

where $d$ is the Euclidean distance and $\zeta$ is a geometrical parameter usually set to $1 / 2$.

The marker points are advected using bilinear interpolation and a redistribution is done at every time step to ensure a uniform distribution as the bubble deforms. The average distance between markers is of the order of the grid size. As underlined by Popinet \& Zaleski (1999) the computational cost of the marker part of the algorithm scales as $1 / n$ where $n$ is the number of grid points along one dimension and is then negligible for reasonable domain sizes.

\subsection{Validation tests}

The time evolution of the radius of a spherically symmetric bubble surrounded by an incompressible fluid is described by the Rayleigh-Plesset equation (Plesset \& Prosperetti 1977). As we are interested in bubbles oscillating radially, it is important to obtain a good agreement between direct numerical simulations and the numerical solution of the Rayleigh-Plesset equation. In the test case illustrated on Figure 4, a bubble with an equilibrium radius $R_{0}=5 \mu \mathrm{m}$ is set in a fluid initially at rest. The initial radius of the bubble is $10 \mu \mathrm{m}$. A constant pressure is applied on three sides of the simulation domain (the fourth side being the axis of symmetry) and the velocity gradient is set to zero on these three sides. The physical parameters are as follows: dynamic viscosity $\mu=0.001$ $\mathrm{kg} / \mathrm{ms}$, surface tension coefficient $\sigma=0.07 \mathrm{~kg} / \mathrm{s}^{2}, \rho=1000 \mathrm{~kg} / \mathrm{m}^{3}, p_{\infty}=10^{5} \mathrm{~Pa}$. The pressure in the bubble is given by a polytropic law of the form $p(R)=p_{0}\left(R_{0} / R\right)^{3 \gamma}$ with 


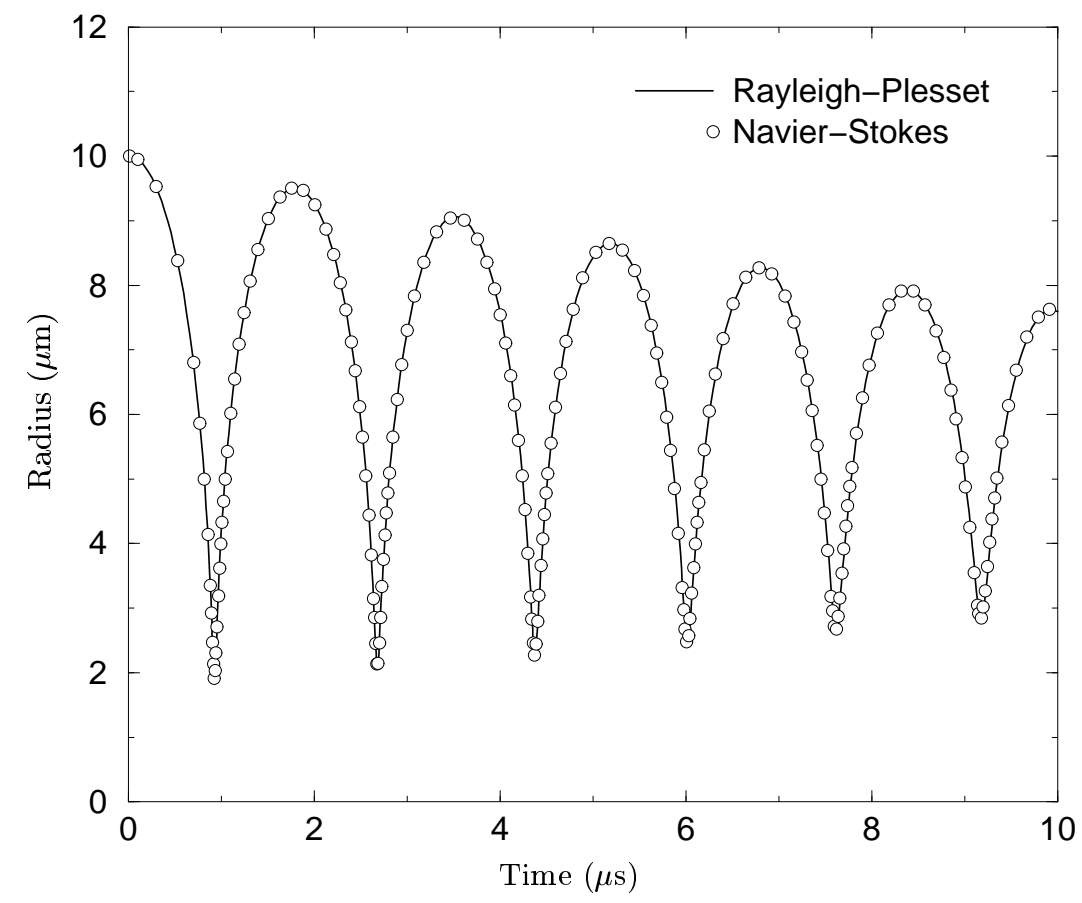

FigURE 4. Free radial oscillations of a bubble of $5 \mu \mathrm{m}$ equilibrium radius.

$\gamma=7 / 5$. The bubble oscillates radially while keeping its spherical shape. The amplitude of the oscillations decreases due to viscous damping. The agreement between the direct numerical simulation and the numerical solution of the Rayleigh-Plesset equation is excellent. The relative quadratic error between the two solutions illustrated is smaller than one percent.

It is important to note that to obtain such an agreement it is necessary to minimize the influence of the boundary conditions in the simulation. This is done by using very large simulation domains. The ratio between the domain size and the bubble diameter is 160. By using the adaptive multidomain technique presented in the following section the computational cost is still reasonable (approximately one hour on a Pentium $350 \mathrm{MHz}$ for a base grid of $128 \times 64$ ).

This simulation is also a good validation test for the extrapolation technique presented in the previous section. The pressure jump on the free-surface due to the normal component of the viscous stress controls the viscous damping of the solution. A $2 \%$ variation in viscosity leads to solutions of the Rayleigh-Plesset equation varying by $1.3 \%$. Given the $1 \%$ error that we obtain, we can conclude that local derivatives of the velocity field near the interface differ by less than $2 \%$.

However this test does not involve any deformation of the interface and the influence of surface tension is limited to a constant pressure jump. A second simple test where the driving force is surface tension is illustrated on Figure 5. A slightly ellipsoidal bubble is set in a liquid initially at rest. Under the influence of surface tension the bubble shape oscillates around its spherical equilibrium position. The parameters are as follows: equivalent radius $R=5 \times 10^{-4} \mathrm{~m}$, surface tension coefficient $\sigma=0.07 \mathrm{~kg} / \mathrm{s}^{2}$, kinematic viscosity $\nu=5 \times 10^{-6} \mathrm{~m}^{2} / \mathrm{s}, \rho=1000 \mathrm{~kg} / \mathrm{m}^{3}$. In order to limit the effect of confinement 


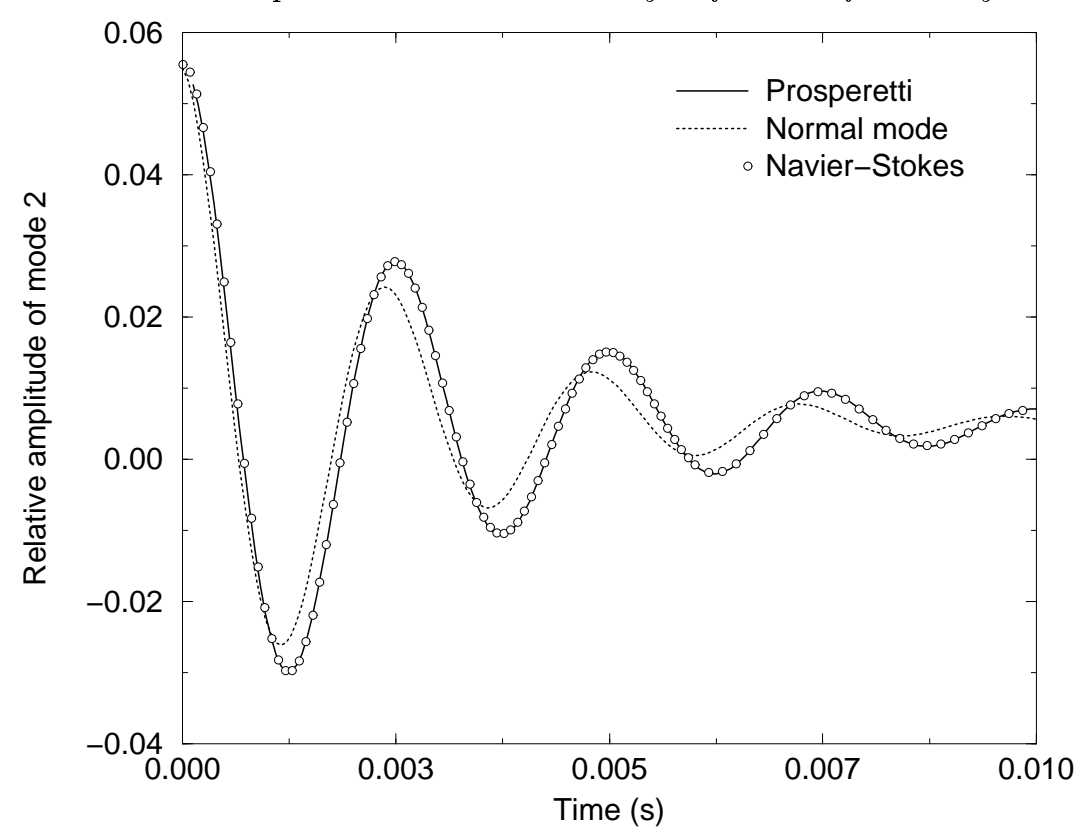

FiguRE 5. Temporal evolution of the second mode of deformation of a slightly ellipsoidal bubble set in a liquid initially at rest.

the ratio between the size of the domain and the bubble diameter is 240 . The diameter of the bubble is about 128 grid points and the multidomain technique is used.

The temporal evolution of the relative amplitude of the second mode of deformation is illustrated together with two theoretical solutions. The first one is the classical normal mode analysis of Lamb (1932). It supposes a stationary regime of oscillation and does not take into account transient effects such as the diffusion of vorticity in the liquid. The second solution is a numerical inversion of a Laplace transform obtained by Prosperetti (1980) taking into account transient effects. This solution is exact for this problem in the limit of a vanishing amplitude of oscillation. The agreement between the numerical simulation and Prosperetti's theory is excellent with a relative quadratic error of $0.4 \%$ for the first eight periods of oscillation. It is also interesting to note that the difference between the approximate normal mode solution and Prosperetti's solution is significant. This test further confirms that vorticity generation at the free surface is accurately described by our interpolation technique.

\section{Comparison with experiments}

In order to assess the applicability of our numerical method to real cases, we wanted to compare our results with experimental measurements. Lauterborn and collaborators have developed an elegant technique to generate highly reproducible bubbles near solid boundaries (Lauterborn \& Bolle 1975; Lauterborn \& Ohl 1997; Philipp \& Lauterborn 1998). The high speed photographic series of Figure 6 illustrate one of these experiments. A focused short laser pulse is fired in water near a solid wall, a gas bubble is then formed and expands, eventually reaches a maximum radius and then collapses violently. A jet is formed near the point of minimum radius and penetrates the re-expanding bubble.

The main problem we are confronted with is the choice of initial conditions for the 
numerical simulation. In fact, neither the initial radius (or initial pressure), nor the equilibrium radius (or gas content) of the bubble are known. We have chosen to use initial conditions given by the fit of the classical Rayleigh-Plesset equation (Plesset \& Prosperetti 1977) to the measured time-evolution of the radius. The experimental time evolution has been measured directly using a digital version of the photographic series and image processing techniques.

The numerical simulation uses a $512 \times 512$ grid. The maximum bubble radius reaches 50 grid points. The bottom boundary of the domain is a free-sliding solid wall (the normal component of the velocity is zero, there is no constraint on the tangential component) and the right boundary is the axis of symmetry. On the top and left walls the pressure is constant and set to the ambient pressure (gravity is neglected). No constraint is imposed on the velocity. The dynamic viscosity for water is $10^{-3} \mathrm{~kg} / \mathrm{ms}$, the surface tension coefficient for an air-water interface $\sigma=0.072 \mathrm{~kg} / \mathrm{s}^{2}$ and the density of the liquid is $1000 \mathrm{~kg} / \mathrm{m}^{3}$. The process is assumed to be adiabatic and the gas pressure is given by $p=p_{0}\left(V / V_{0}\right)^{\gamma}$ where $V$ is the volume of the bubble, $V_{0}=4.78 \times 10^{-9} \mathrm{~m}^{3}, p_{0}=100072$ $\mathrm{Pa}$ and $\gamma=7 / 5$. The surrounding fluid is initially at rest and the initial radius of the bubble is $0.4 \mathrm{~mm}$.

Simulation results are shown on Figure 6 with the same spatial layout and interframe time. Figure 7 illustrates the temporal evolution of the equivalent radius (defined as $3 V^{1 / 3} / 4 \pi$ ) together with the evolution measured experimentally and given by the Rayleigh-Plesset calculation. The agreement between the experiment and the numerical result is relatively good with initial jet formations qualitatively and quantitatively similar. Both the experiment and the simulation clearly show the jet impacting and deforming the opposite side of the bubble, while the jet is stretched by the bubble expansion. The jet velocity is well reproduced by the numerical model, the tip of the deformed bubble touching the wall at approximately the same time. Another interesting feature revealed by the numerical simulation is the "splashing" effect of the jet impact with the formation of an axisymmetric rim expanding with the bubble (Figure 8). This observation is very similar to results reported by Blake et al. $(1997,1998)$ using a boundary integral technique. 
Bubble collapse near a solid boundary: influence of viscosity
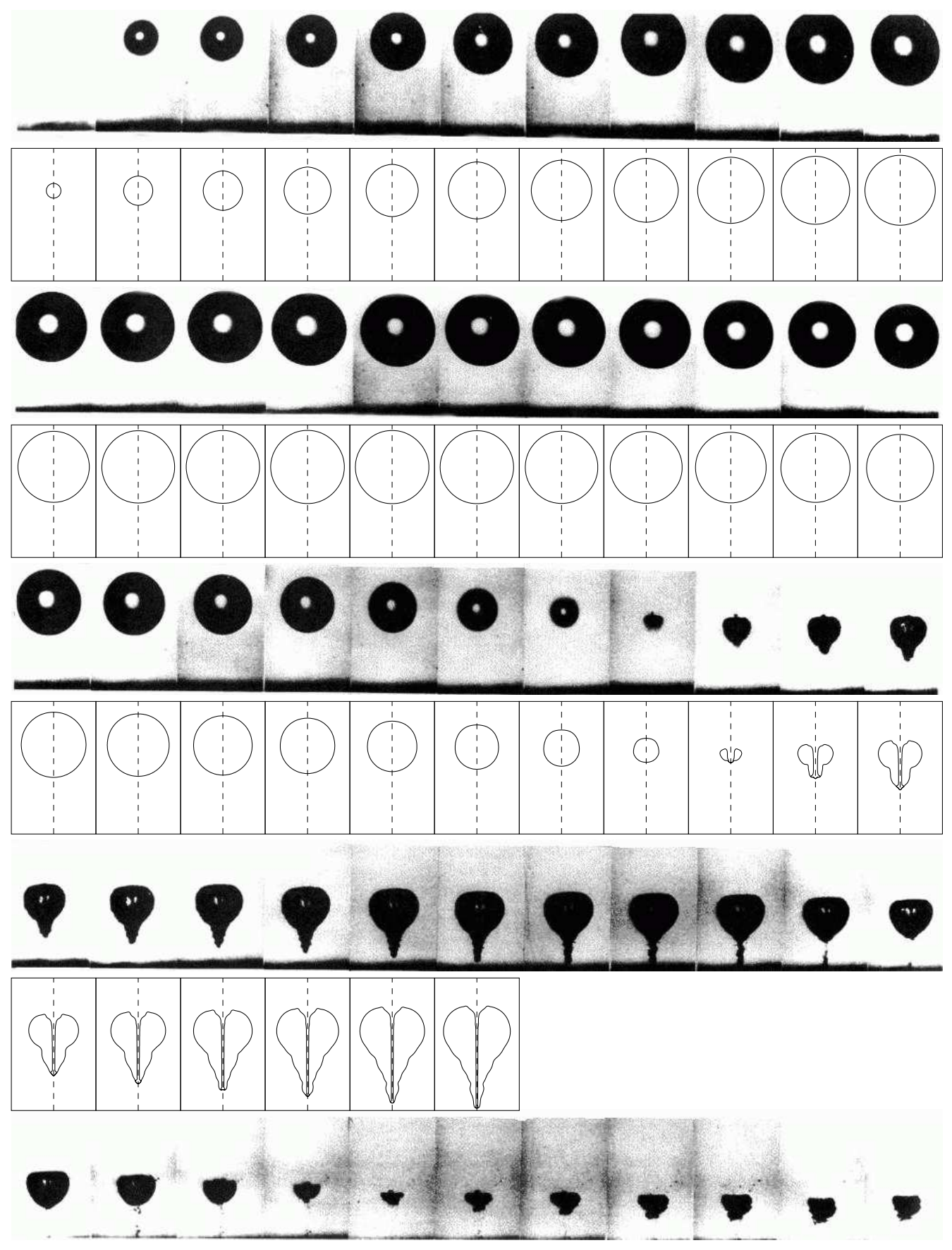

FIGURE 6. Comparison between a high-speed photographic series of a bubble collapsing near a wall (Lauterborn \& Ohl 1997) and a direct numerical simulation. Sampling rate is 75000 frames per second. 


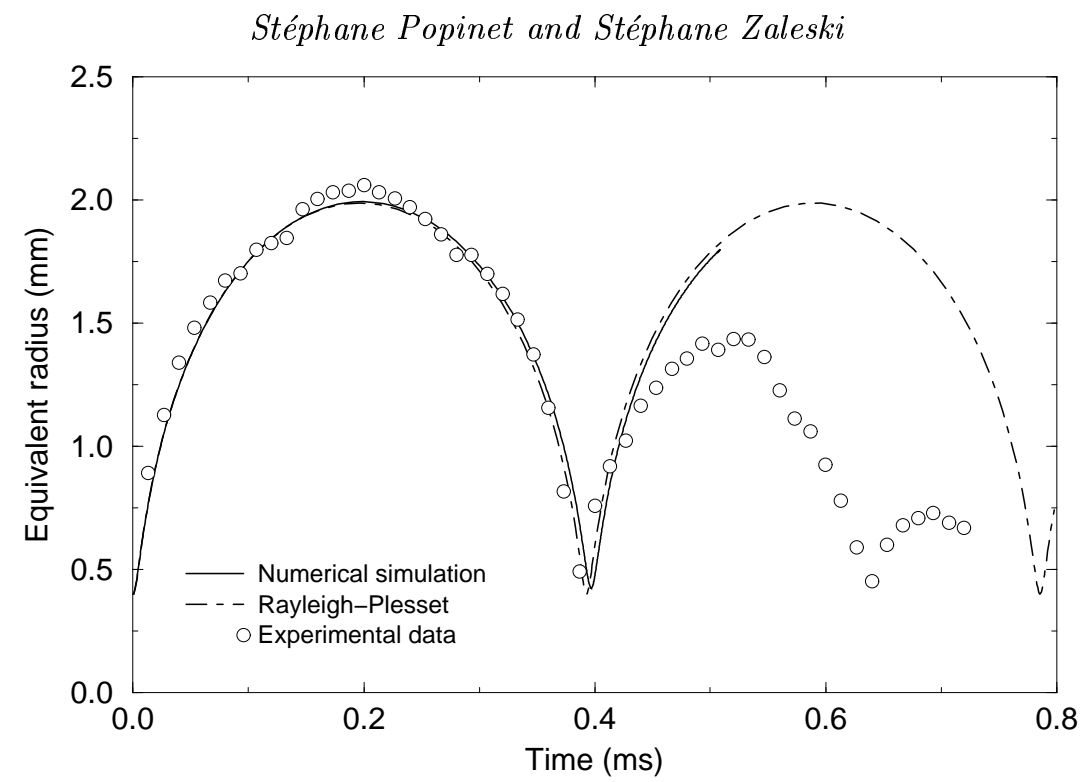

Figure 7. Temporal evolution of the equivalent radius of the bubble as given by experimental measurements, numerical simulation and the Rayleigh-Plesset equation.

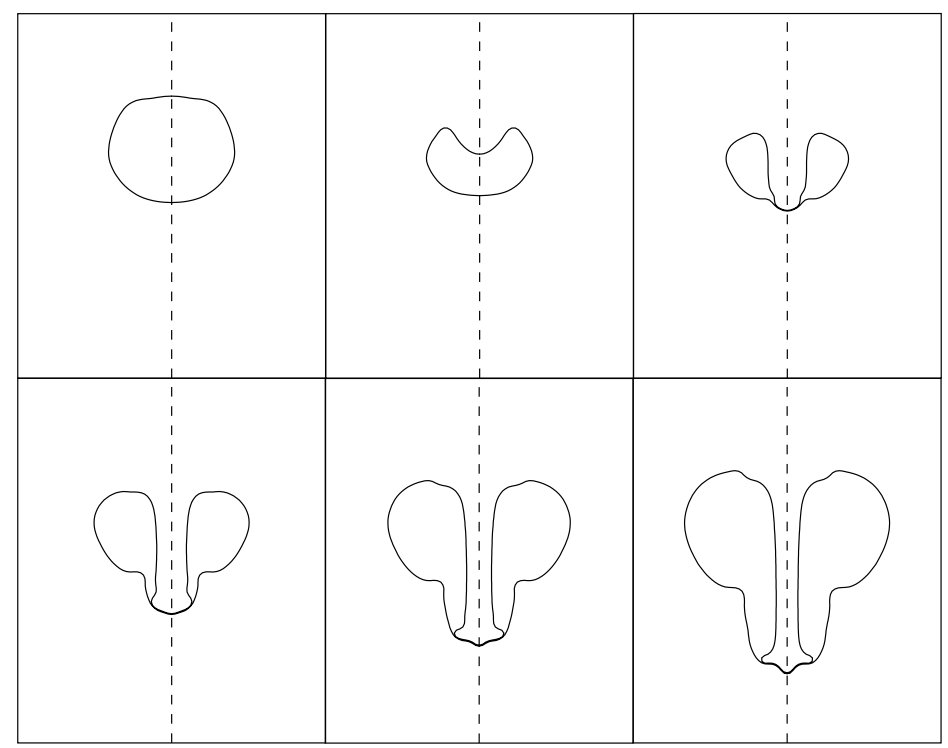

FiguRE 8. Jet formation and impact. 
However, we can see both on Figures 6 and 7 that the rebound of the bubble is much larger in the numerical simulation than that observed in the experiment. The RayleighPlesset equation gives a result comparable to the numerical calculation. This would indicate that the effects of fluid compressibility, in particular the emission of acoustic and shock waves, which are not taken into account by our incompressible code or by the Rayleigh-Plesset equation, are an important source of energy dissipation. Moreover, the very simple equation of state chosen to describe the gas contained in the bubble does not take into account heat transfer and phase change at the interface between the liquid and the gas.

\section{Numerical study of the influence of viscosity on jet formation and evolution}

While interesting for validation purposes, the previous result is of limited interest due to the small influence of viscosity on the fast collapse of relatively big bubbles. For this type of simulation, a boundary integral code with a viscous boundary layer approximation would probably give good results (Plesset \& Chapman 1971; Blake et al. 1993). For this reason, we have chosen to focus our attention on the influence of viscosity on jet formation and evolution for moderate Reynolds numbers.

In order to study the influence of viscosity, we first need to find which characteristic parameters control the problem. We assume that the pressure in the gas is described by a polytropic law of the type

$$
p(R)=p_{\infty}\left(\frac{R_{0}}{R}\right)^{3 \gamma},
$$

where $R$ is the radius of the bubble, $R_{0}$ is the equilibrium radius, $p_{\infty}$ is the ambient pressure and $\gamma$ is a polytropic exponent. We can chose $R_{0}$ as a length scale and $\sqrt{\rho R_{0}^{2} / p_{\infty}}$ as a time scale. The Rayleigh-Plesset equation (Plesset \& Prosperetti 1977) describes the evolution of the radius and can be written in non-dimensional form as

$$
R_{\star} \ddot{R}_{\star}+\frac{3}{2} \dot{R}_{\star}^{2}+4 \nu_{\star} \frac{\dot{R}_{\star}}{R_{\star}}=R_{\star}^{-3 \gamma}-1-\frac{2 \sigma_{\star}}{R_{\star}},
$$

where the $\star$ denotes non-dimensional quantities. The set of characteristic coefficients is then

$$
\nu_{\star}=\frac{\nu}{R_{0}} \sqrt{\frac{\rho}{p_{\infty}}}, \quad \sigma_{\star}=\frac{\sigma}{R_{0} p_{\infty}} \text { and } \gamma
$$

where $\sigma$ is the surface tension coefficient, $\nu$ the kinematic viscosity and $\rho$ the density. We need to add the coefficients characteristic of the initial conditions. The relative initial radius $\alpha=R_{M} / R_{0}$ and the relative initial distance from the boundary $\beta=H / R_{M}$, where $H$ is the distance from the center of the bubble to the solid wall. Thus we have five independent parameters: $\alpha, \beta, \gamma, \nu_{\star}, \sigma_{\star}$. In order to simplify the study, we will neglect surface tension $\left(\sigma_{\star}=0\right)$ and assume that the gas is diatomic and the process adiabatic $(\gamma=7 / 5)$. A Reynolds number is defined as $R e=1 / \nu_{\star}$.

In order to resolve the small scale which can occur for high compression ratios correctly we have used an adaptive hierarchy of grids. Each grid has a fixed number of points $(128 \times 64$ in all the results illustrated here) but is half the size of its parent. A typical setup is shown on Figure 9. Grids are added or removed as the bubble shrinks and grows. Moreover, this technique allows the use of relatively large simulation domains in order to minimise the influence of the boundary conditions.

Figures 10, 11 and 12 illustrate the influence of the Reynolds number on jet formation 


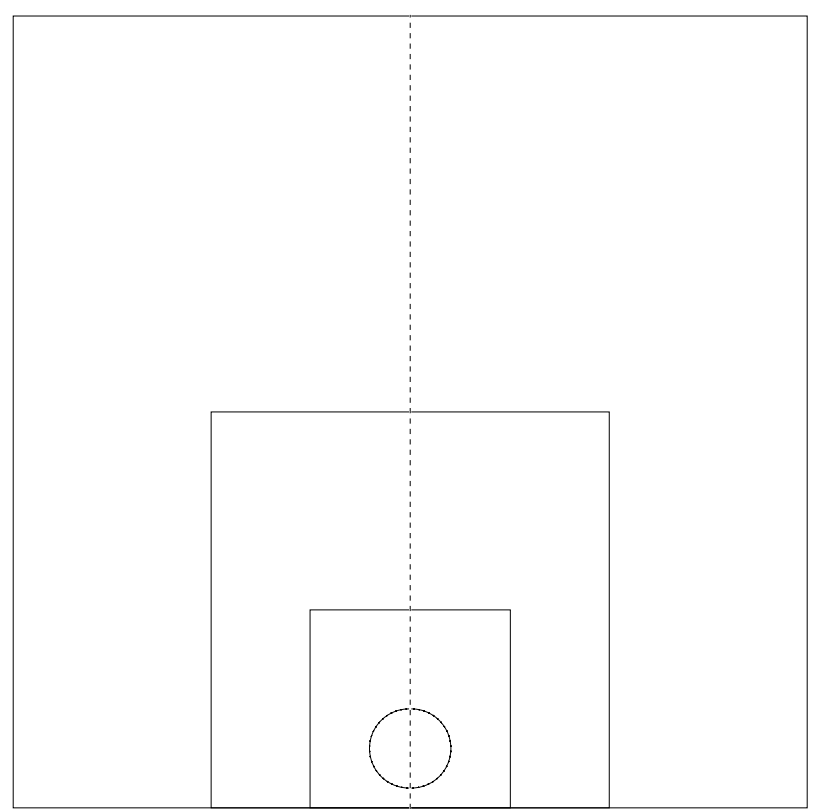

FigurE 9. Example of the hierarchy of grids used to deal accurately and efficiently with large variations in bubble radius.

and impact velocity. For high Reynolds numbers (Figure 10) the initial jet velocity is high and the impact occurs shortly after jet formation. For low Reynolds numbers (Figure 12), the initial jet velocity is small and the velocity of rebound of the opposite wall of the bubble is large enough to prevent any impact. For intermediate Reynolds numbers (Figure 11), the initial velocity of the jet is comparable to the velocity of rebound of the opposite wall, the jet is stretched by the expansion of the bubble, the impact is delayed and the impact velocity is small. The critical Reynolds number $R e_{c}$ is defined as the Reynolds number for which the impact velocity is zero. If the Reynolds number is smaller than $R e_{c}$, the jet never impacts the other side of the bubble.

This transition is illustrated differently on Figures 13 and 14. The lower curves (thick lines) represent the time evolution of the position of the south pole (closer to the wall) for the different Reynolds numbers shown in the legend. The thin lines are the time evolution of the north pole and the upper curves describe the evolution of the position of the point farther away from the solid wall. The upper and lower curves describe the global dynamics of the bubble: collapse until $t \approx 2$ followed by a re-expansion and motion of the center of gravity toward the wall. The thin lines describe the jet dynamics. Initially there is no jet and these curves are indistinguishable from the upper curves. At the time of curvature inversion occurring at the north pole and corresponding to jet formation the curves separate (Figure 14). The jet continues to penetrate inside the bubble and eventually hits the other side. If viscosity is too high the jet is slowed down and never makes it to the south pole.

As can be seen on Figure 11, when $R e$ is close to $R e_{c}$ the jet becomes very thin and the impact occurs late in the cycle of oscillation. If we want to find the value of $R e_{c}$ with a reasonable accuracy, we need to use a very fine grid in order to model correctly the flow inside the jet. Such high resolutions would be impractical for a parametric study of $\operatorname{Re}_{c}(\alpha, \beta)$. We therefore sought an alternative and more easily computable definition of $R e_{c}$. 


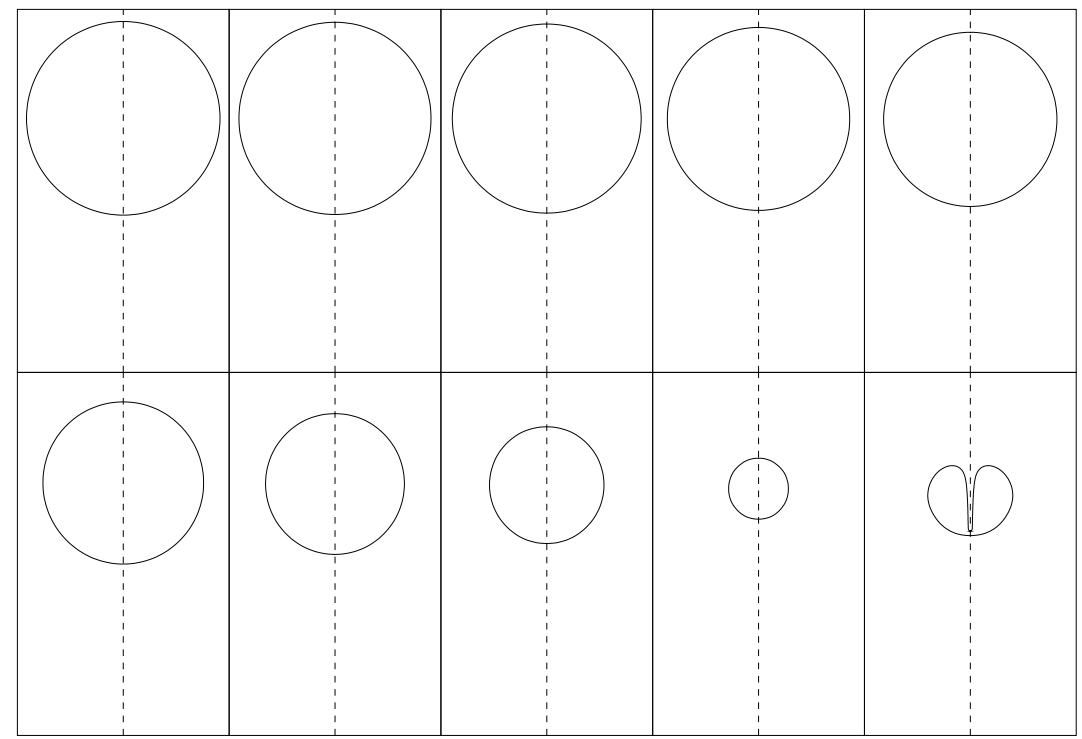

Figure 10. Time evolution of a bubble collapsing near a wall at high Reynolds number. The wall is along the bottom side of the box. Not all the simulation domain is shown. Interframe time is 0.243 . $R e=29.906, \alpha=2.023, \beta=2.625$.

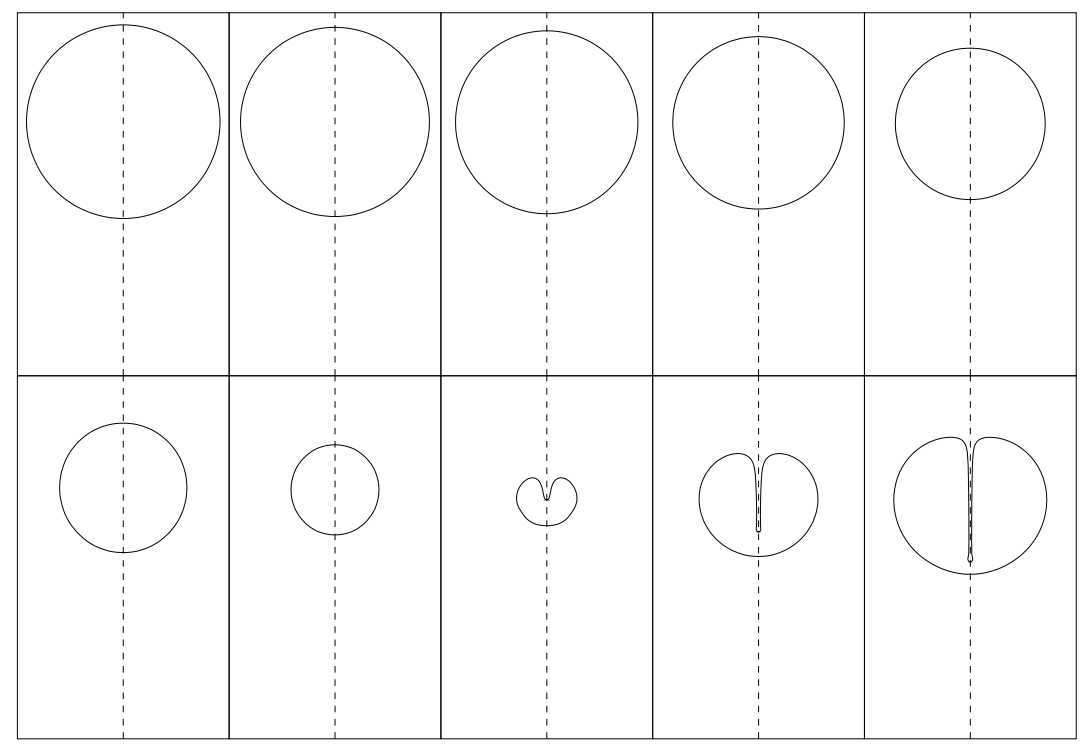

Figure 11. Time evolution of a bubble collapsing near a wall at intermediate Reynolds number. Interframe time is 0.303 . $R e=24.519, \alpha=2.023, \beta=2.625$.

Figure 15 illustrates the time evolution of the relative velocity between the top and bottom poles for various values of $R e$. The relative velocity is chosen to be positive during collapse and negative during expansion. The black dots indicate the instant of impact and the white dots indicate the instant of jet formation (curvature changes sign at the top pole). As the Reynolds number decreases the impact is delayed and the impact velocity decreases. For values of $R e$ close to $R e_{c}$, the curve has two extrema: a maximum relative velocity is reached near the end of the collapse and a minimum relative velocity occurs 


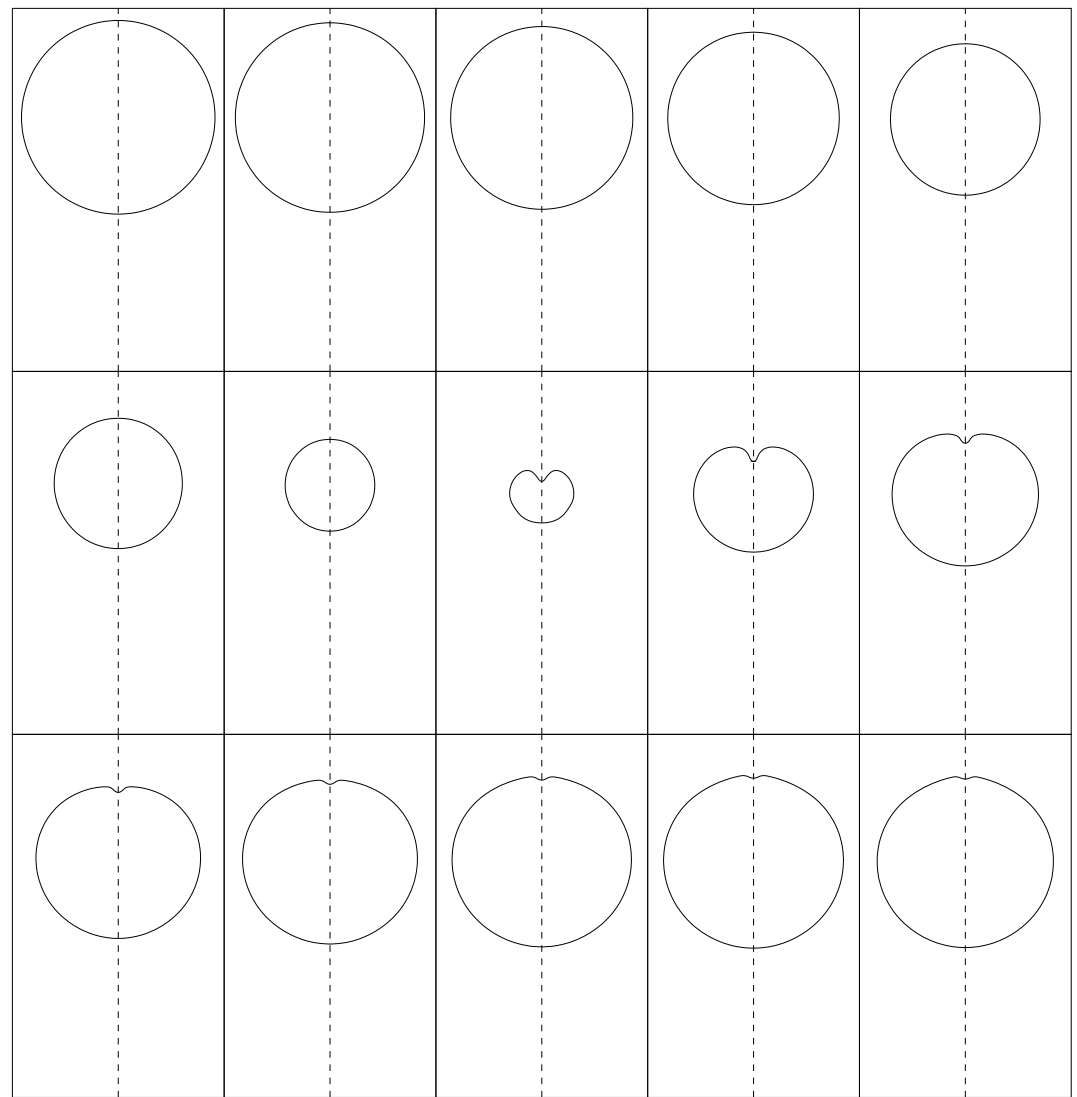

Figure 12. Time evolution of a bubble collapsing near a wall at low Reynolds number. $R e=18.413, \alpha=2.023, \beta=2.625$.

not long after jet formation. Moreover, near the critical Reynolds number, the relative velocity at impact is seen to be close to its minimum value. Therefore we take the value of $R e$ for which the minimum relative velocity is zero as an alternative definition of $R e_{c}$ (i.e. at some point close to the beginning of jet formation the tip of the jet is moving exactly as fast as the bubble is re-expanding).

Figure 16 confirms that both definitions give close values for $\operatorname{Re}_{c}$ (17.15 and 17.77 for the impact and the minimum relative velocity respectively). Moreover, the impact velocity is strongly dependent on the Reynolds number near the critical value. This new criterion is much easier to test numerically since we only need the value of the minimum velocity and do not need to solve the jet impact directly.

The curves on Figure 17 have been obtained for various values of $\alpha$ (shown in the legend). Each value of $R e_{c}$ is found using a bisection technique. On average, five simulations are necessary to find a value of $R e_{c}$ with a $3 \%$ accuracy (each simulation takes approximately fifteen minutes on a $\mathrm{PC}$ ). As the value of $\beta$ increases, it becomes more difficult to form a jet and viscous effects play a more important role: the value of $R e_{c}$ increases. On the other hand when $\alpha$ increases, the collapse velocity is larger and viscous effects tend to be smaller: the value of $R e_{c}$ decreases.

The curves of Figure 17 can be rescaled as shown on Figure 18. The black curves on Figure 17 are the rescaled versions of the interpolating curve of Figure 18. The agreement is excellent, and the discrepancies observed in particular for high values of $\beta$ for $\alpha=2.023$ 


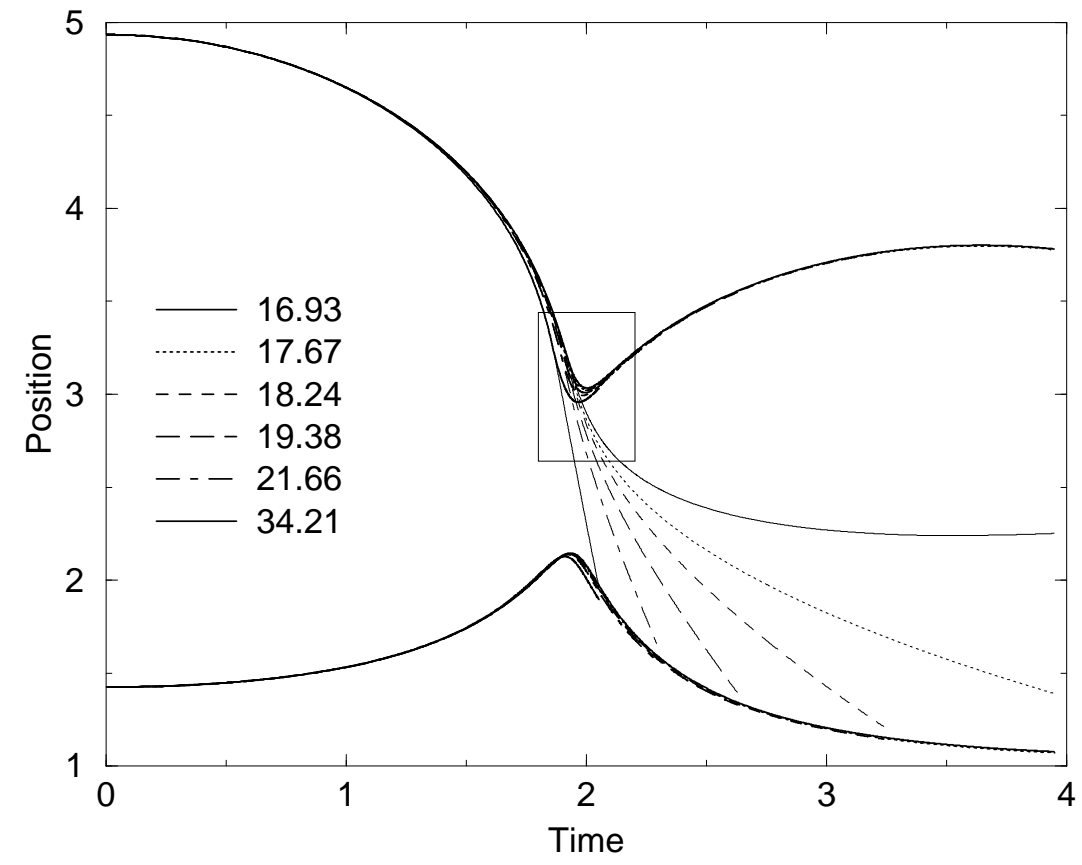

FIgURE 13. Time evolution of the position of the poles as a function of the Reynolds number indicated in the legend. $\alpha=1.754, \beta=1.5$.

and $\alpha=1.921$ can be attributed to the formation of thin jets which are difficult to resolve accurately.

We have obtained this scaling empirically by measuring numerically the correlation $\mathcal{C}(\eta, \zeta)$ between the transformed point sets, where the transformation is: $\beta \rightarrow \beta / \alpha^{\eta}$ and $R e_{c} \rightarrow R e_{c} / \alpha^{\zeta}$. As illustrated on Figure 19, the maximum correlation is obtained for values of $\eta$ and $\zeta$ close to 3 and $1 / 2$ respectively. 


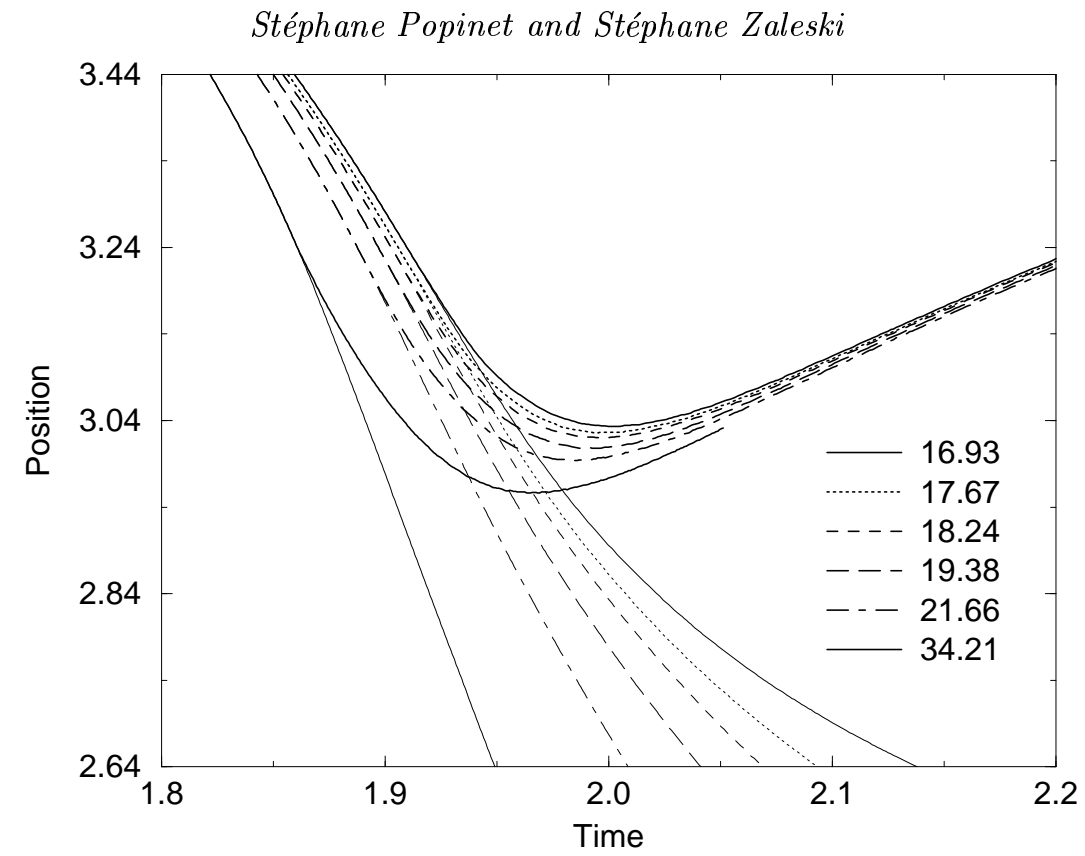

Figure 14. Detail of Figure 13.

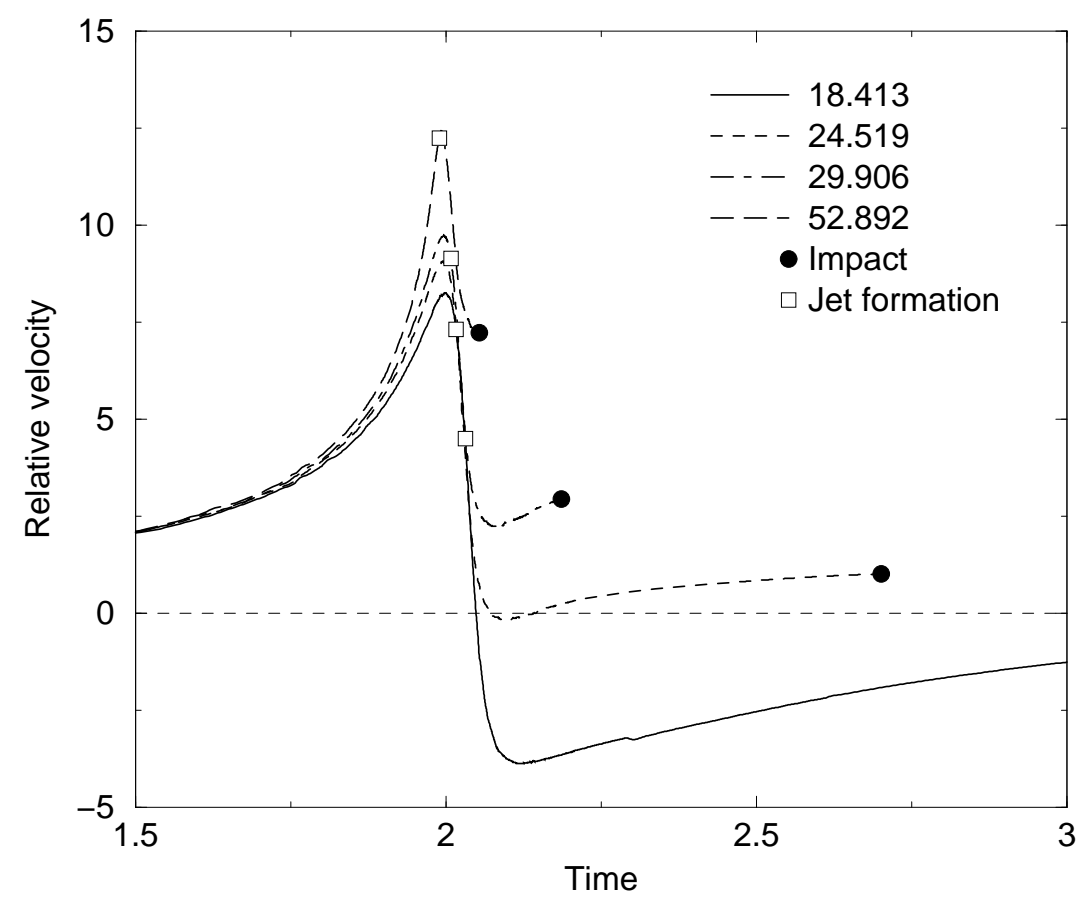

FIGURE 15. Relative velocity between the top and bottom pole of a collapsing bubble as a function of time for various values of the Reynolds number (indicated in the legend). The white squares and black dots mark the time of jet formation (curvature changes sign) and jet impact respectively. $\alpha=2.023, \beta=2.625$. 


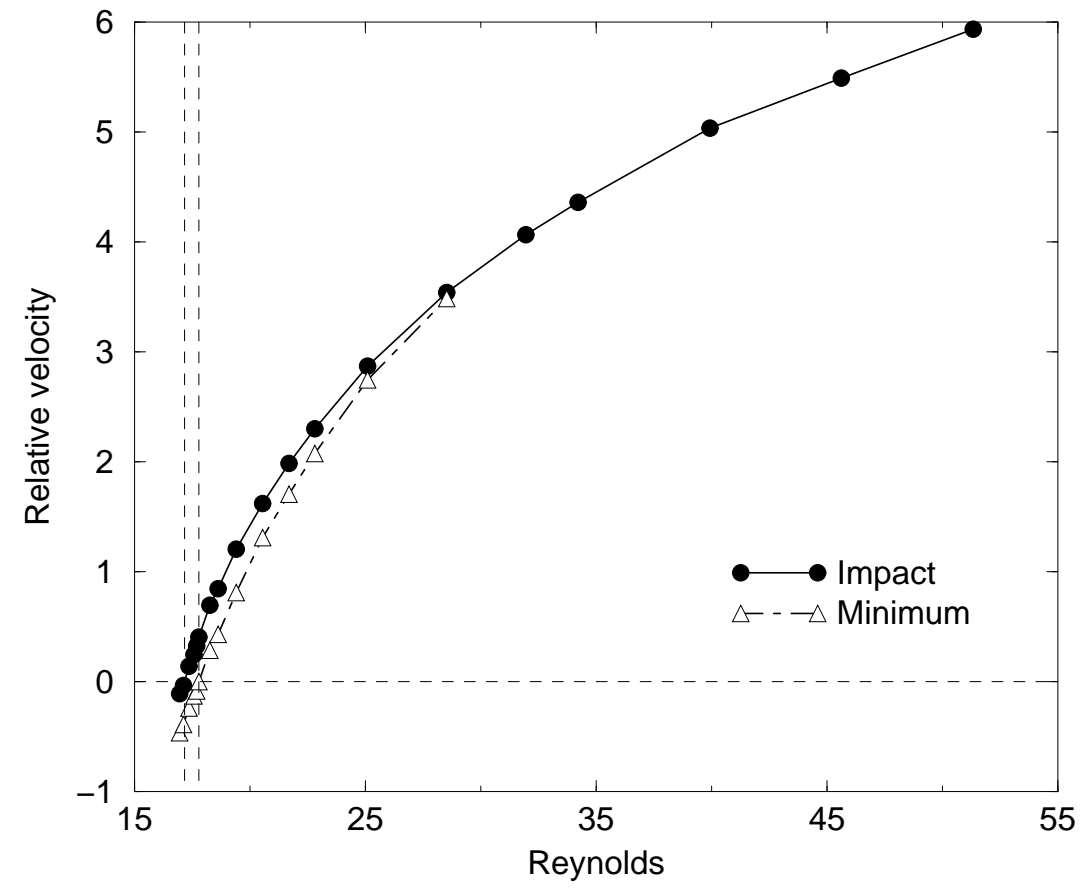

FIGURE 16. Relative impact velocity and minimum of the relative velocity as a function of the Reynolds number. Taking as critical Reynolds number the value of the Reynolds for which the minimum relative velocity is zero gives a good approximation of the Reynolds number for which the jet impact velocity is zero. $\alpha=1.754, \beta=1.5$. 


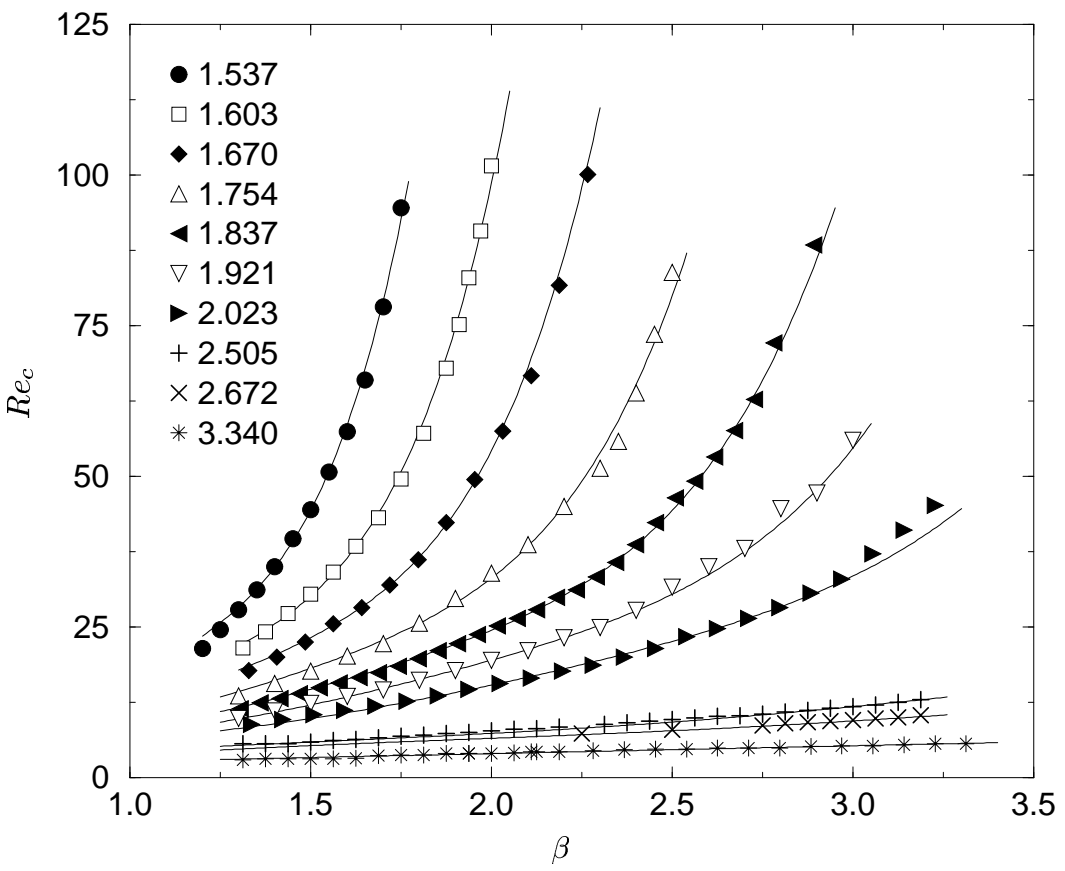

FIgURE 17. Value of the critical Reynolds number $R e_{c}$ as a function of the relative distance to the solid wall $\beta$. Each curve corresponds to a different value of the relative initial radius $\alpha$ (indicated in the legend).

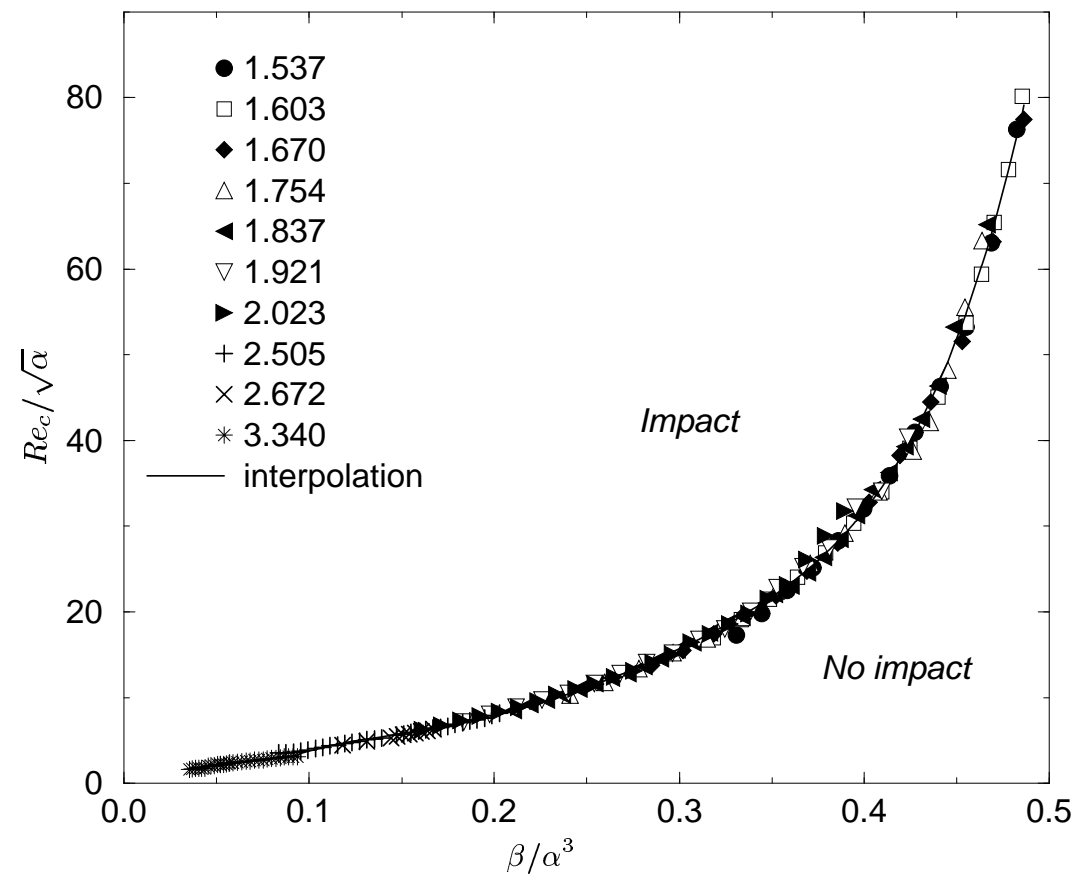

Figure 18. Rescaled version of Figure 17. The black line is an interpolating polynomial. 

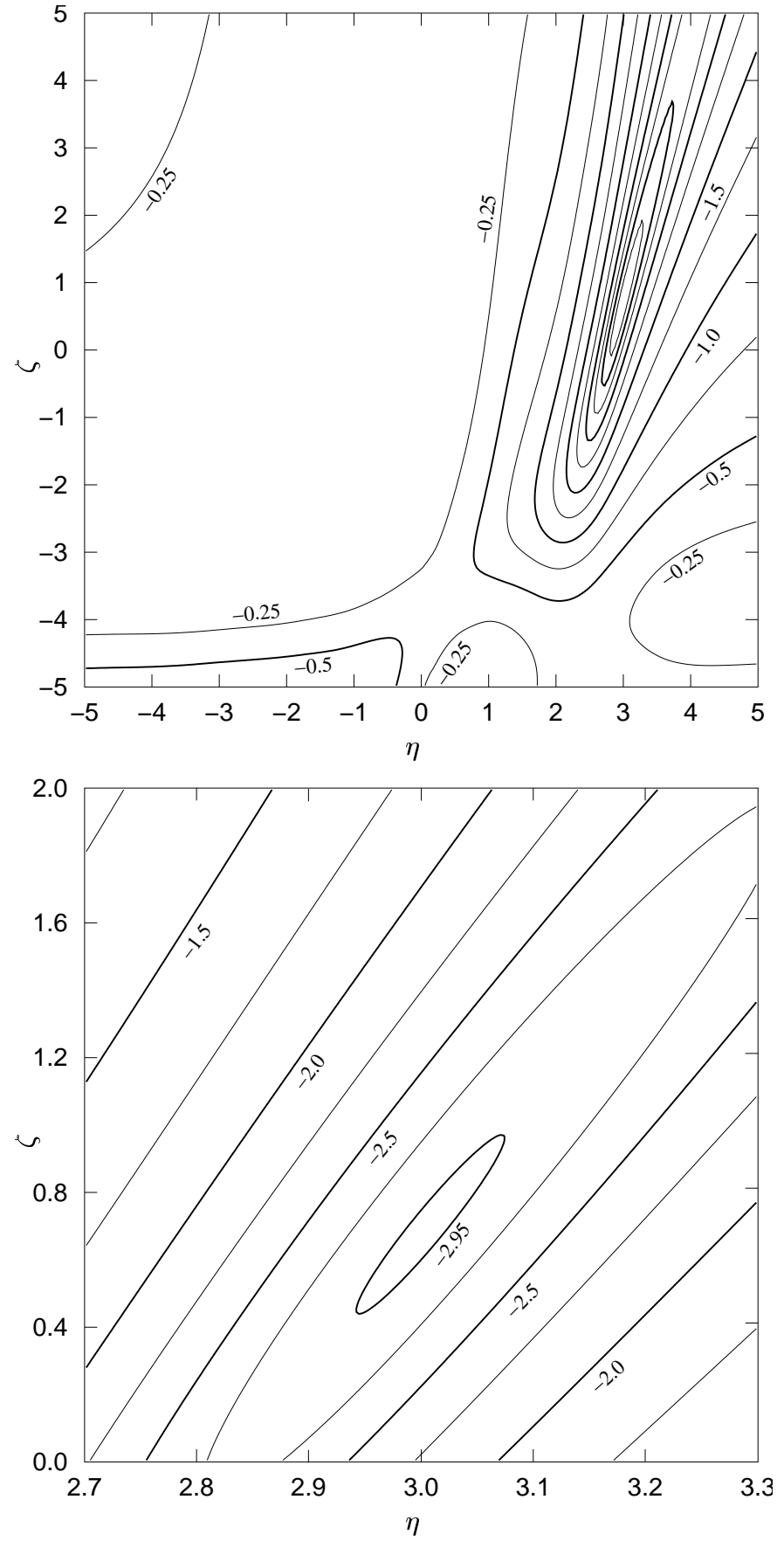

Figure 19. Numerical evaluation of the correlation $\mathcal{C}(\eta, \zeta)$ between the rescaled point sets of Figure 17. 


\section{Theory}

In what follows we describe a theory based on the approximation of a relatively large Reynolds number and a large initial bubble radius $\alpha$ and distance to the wall $\beta$. Thus in our estimates we first neglect the viscous effects. Then we discuss stability and viscous corrections.

\subsection{Velocity condition}

The asymmetrical bubble collapse that heralds jet formation is influenced by the presence of the wall. While the approximately spherical bubble shrinks in size, its center of mass is attracted towards the wall by the image bubble. As the bubble approaches the wall, a momentum $Q$ is gained mostly by the liquid phase surrounding it, the so-called added mass momentum. In equations

$$
\dot{Q}=-\frac{4}{3} \pi R^{3} \frac{\partial p}{\partial z}
$$

where we have used the dimensionless variables as in (4.2) but where as in what follows we dropped the $\star$ subscripts for simplicity. The added-mass momentum may be expressed in terms of the vertical position of the bubble $z_{b}(t)$ as

$$
Q=C_{M} \frac{4}{3} \pi R^{3} \dot{z}_{b}
$$

where $C_{M}$ is the added mass coefficient, approximately $C_{M}=1 / 2$ at high Reynolds numbers. To estimate conditions for jet formation and impact, we distinguish two effects: the stability or instability of the near-spherical bubble motion, and the possible mismatch between the time scale of the rebound and the time scale of the jet traversing the bubble. The first condition is a jet formation condition, while the second is a sufficient velocity condition. We begin with the jet impact.

In what follows, we first investigate the spherical collapse, then the effect of the image bubble. We neglect viscosity. As in the numerics, surface tension is left aside. Then Equation (4.2) becomes

$$
R \ddot{R}+\frac{3}{2} \dot{R}^{2}=R^{-3 \gamma}-1
$$

This equation integrates to

$$
E_{0}=R^{3} \dot{R}^{2}+\frac{2}{3 \gamma-3} R^{-3 \gamma+3}+\frac{2}{3} R^{3}
$$

We have $R=R_{m}$ at minimum radius and $R=\alpha$ at maximum radius. Assuming $\alpha \gg 1$ we have $E_{0}=\frac{2}{3} \alpha$ and

$$
R_{m} \sim[(\gamma-1) \alpha]^{\frac{1}{1-\gamma}}
$$

This formula is a good approximation for high $R e$ as confirmed by Figure 20 where we have plotted the ratio of the maximum to minimum radius $\alpha / R_{m}$ for different values of $\alpha$ and $R e$. All the simulations we have performed are represented which also shows the small influence of $\beta$ on the compression ratio.

In what follows we omit $\gamma$-dependent prefactors. A full solution may be found for the inviscid motion in the form of an integral, but we shall only need some basic asymptotic features of the solution at large $\alpha$. We have a time scale during which the radius remains close to $R_{m}$ and the typical acceleration is $\ddot{R}_{m}$. From (5.3)

$$
\ddot{R}_{m} \sim R_{m}^{-3 \gamma-1}
$$




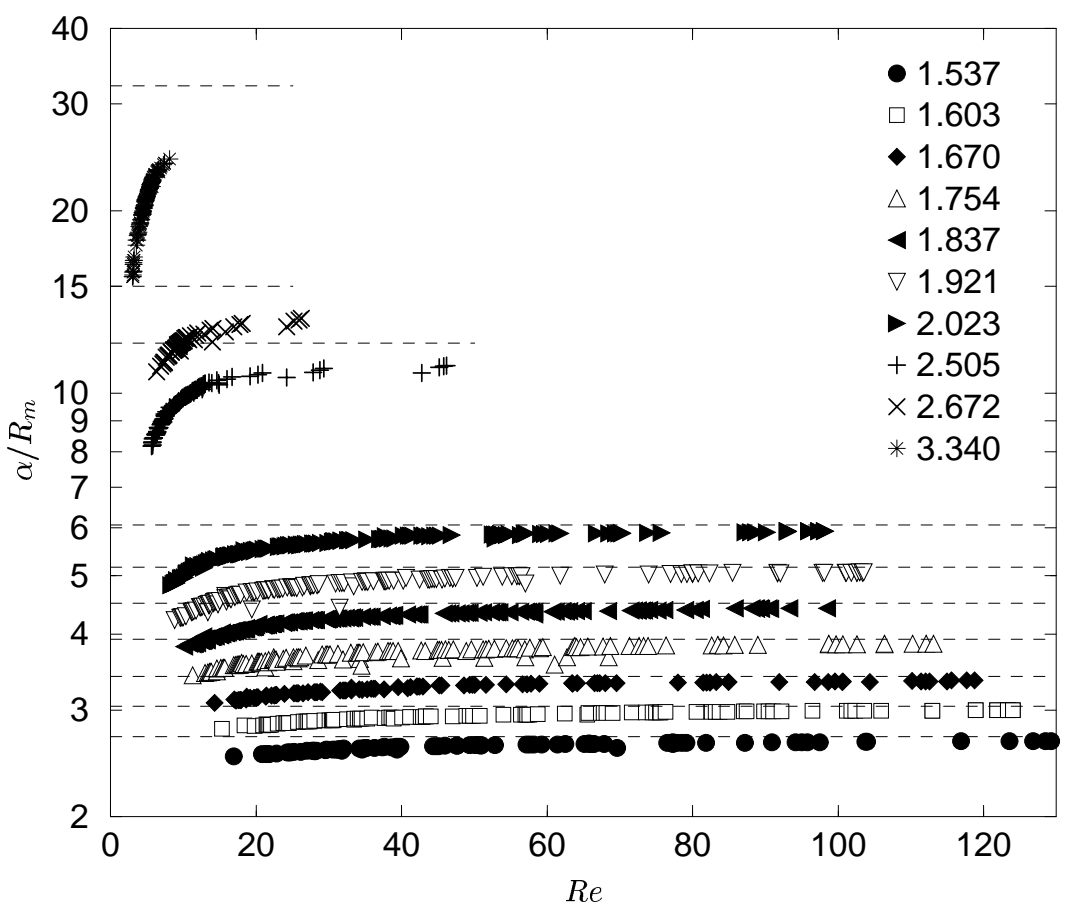

FIGURE 20. Ratio between the maximum (initial) radius $\alpha$ and the equivalent minimum radius $R_{m}$ as a function of the Reynolds number and for the different values of $\alpha$ indicated in the legend. Theoretical values in the inviscid case given by equation (5.5) are represented by the dashed lines.

and thus

$$
t_{m} \sim \alpha^{\frac{2+3 \gamma}{2-2 \gamma}}, \quad \ddot{R}_{m} \sim \alpha^{\frac{3 \gamma+1}{\gamma-1}} .
$$

We set the reference time $t=0$ at the point of minimum radius. There is the well-known outer solution for $|t| \sim 1$ of the form

$$
R \sim|t|^{2 / 5} \alpha^{3 / 5}
$$

and an inner solution for $|t| \lesssim t_{m}$. We are now in position to integrate the added-mass equation (5.1). The pressure gradient may be estimated from the pressure field associated with the image bubble. From Bernouilli's equation the dominant term is, in dimensionless units

$$
p=-\frac{1}{2} \frac{\dot{R}^{2} R}{r}-\frac{1}{2} \frac{\dot{R}^{2} R^{4}}{r^{4}} .
$$

At large $\beta$ and at distance $H$ (still in dimensionless units) from the wall the leading order is

$$
|\nabla p| \sim \frac{\dot{R}^{2} R}{H^{2}} .
$$

Thus

$$
Q \sim \int_{-\alpha}^{0} \frac{\dot{R}^{2} R^{4}}{H^{2}} d t .
$$

Using the inner and outer scalings, we find that the leading order contribution comes 
from the outer solution (5.8), thus, since $\beta=H / \alpha$ we get

$$
Q \sim \alpha^{3} \beta^{-2} \text {. }
$$

The jet forms at time 0 near minimum radius. A kind of equipartition principle leads to assume that it gets half the added mass momentum, on a spatial scale of the order of $R_{m}$. Thus the jet velocity $V_{J}$ is given by

$$
V_{J} \sim Q / R_{m}^{3}
$$

and thus

$$
V_{J} \simeq C_{J} \alpha^{\frac{3 \gamma}{\gamma-1}} \beta^{-2}
$$

We have measured the jet velocity directly from the data: the results, although relatively noisy are consistent with the above scaling for $R e>100$ with a prefactor $C_{J} \simeq 0.05$. We can now state our first condition for the impact of the jet: the time that the jet takes to traverse the bubble must be shorter than the small time scale $t_{m}$. For if it were otherwise, the south and north pole of the bubble would separate ever faster, while the jet, due to viscous effect, would slow down. In equations, the critical velocity is of the order

$$
V_{J} \sim R_{m} / t_{m},
$$

which leads to

$$
\beta^{2} \alpha^{\frac{-3}{2} \frac{\gamma}{\gamma-1}} \sim 1
$$

A $\gamma$-dependent numerical constant is bound to appear on the right hand-side. For $\gamma=7 / 5$ we thus have

$$
\beta \alpha^{-21 / 8}=C_{0}
$$

In a graph having $\beta \alpha^{-21 / 8}$ on the horizontal axis and $R e / \alpha^{1 / 2}$ on the vertical axis, the inviscid dynamics yield a vertical line, to the right of which there is no jet impact.

\subsection{Viscous effects}

What happens when a small viscosity is added? Most of our estimates involve only the autonomous dynamics, without the image bubble forcing. All these estimates are affected by corrections of the form $\nu f(\alpha)$ were $f(\alpha)$ is some $\alpha$-dependent expression. The exact expressions are complex since they involve the integrals of the inviscid motion. However the added-mass momentum remains proportional to $\beta^{-2}$, except for the fact that the pressure gradient could now involve additional terms. However, the fluid velocity around the bubble, imposed by incompressibility, is $u=\dot{R} R^{2} / r^{2}$ on which the viscous term $\nu \nabla^{2} u$ vanishes (viscosity comes about in the Rayleigh-Plesset equation only because of surface terms). Thus the pressure field created by the image bubble remains the one computed. Keeping now $\gamma=7 / 5$, all the corrections amount to

$$
\beta \alpha^{-21 / 8}=C_{0}+\frac{f(\alpha)}{R e}+\mathcal{O}\left(\frac{1}{R e^{2}}\right) .
$$

Thus the impact condition, dependent on three variables $\alpha, \beta$ and Re may be collapsed onto a single graph in the variables $x=\beta \alpha^{-21 / 8}$ and $y=R e / f(\alpha)$. In that graph the impact condition asymptotes to the vertical inviscid condition, in a manner consistent with our numerical finding (Figure 21). In the numerical data, $f(\alpha) \simeq \alpha^{1 / 2}$ provides a good fit (although there is some uncertainty on the exponent, see Figure 19). At this time we have not found a convincing theoretical argument yielding $f(\alpha)$. It is possible that $f(\alpha)$ combines several effects that yield an effective scaling law in the range of $\alpha$ considered. 


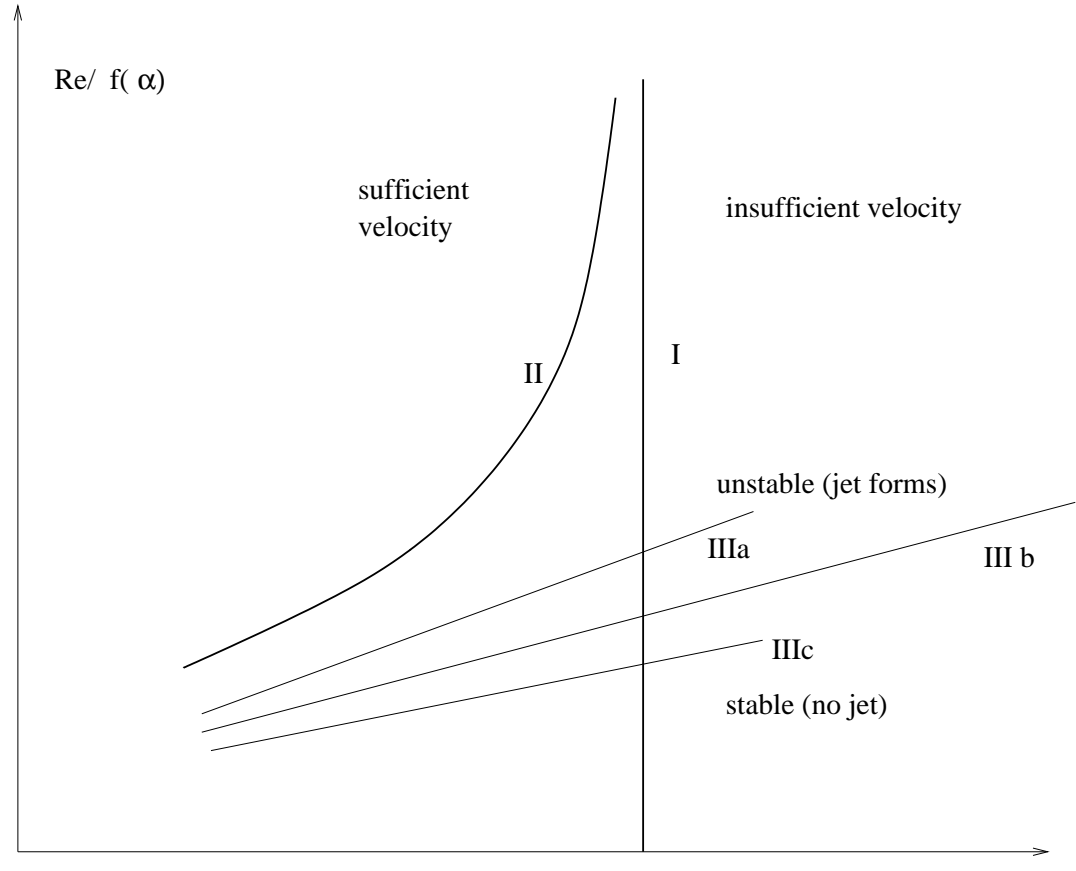

$\beta / \alpha^{21 / 8}$

FIGURE 21. A graphical summary of our scaling theory. Curve I is the asymptotic limit at small viscosity, separating a region where the velocity of the jet is too small for impact from a region of large enough velocity. With viscosity, the separation appears as curve II. Jet formation is possible if there is instability, it occurs above curves IIIa,b,c. However these curves are not scaling with $\beta$, thus each $\beta$ yields a different curve. From our numerical results, there is good reason to assume that all curves of type III are below curve II, so the stability condition is not relevant.

\subsection{Jet formation condition}

The jet formation arises through an instability of the Rayleigh-Taylor type: as the bubble wall is accelerated towards the heavier, liquid phase, it becomes instable to deviations from sphericity. Viscosity may prevent that instability provided that the time scale for viscous diffusion $t_{v}=R_{m}^{2} / \nu$ is smaller than the time scale associated with the RayleighTaylor instability. For plane waves of wave number $k$ the growth rate $s$ is $s=(g k)^{1 / 2}$. Here the most dangerous mode has $k \sim 1 / R_{m}$ and $g$ is $\ddot{R}_{m}$ so the time scale of the instability is $t_{m}$. The condition $t_{m} \sim t_{v}$ then yields $t_{g}=\left(R_{m} / \ddot{R}_{m}\right)^{1 / 2}$.

Equating the viscous and acceleration time scales $t_{g}$ and $t_{\nu}$ yields a critical condition for jet formation:

$$
R e \sim \alpha^{\frac{3 \gamma-2}{2-2 \gamma}} .
$$

In the diatomic case $\gamma=7 / 5$ this yields $R e=\alpha^{-11 / 4}$. Notice that scaling does not involve $\beta$ (the influence of $\beta$ on the exponential growth phase of the instability yields logarithmic corrections.). Thus in the variables of our scaling diagram, retaining the theoretical value of the previous section for the exponent of $x, x=\beta \alpha^{-21 / 8}$ and $y=\frac{R e}{\alpha^{1 / 2}}$ we have $y=(x / \beta)^{22 / 21}$. This is almost a straight line and may have some connection with the lower part of our numerical-experimental curve.

\subsection{Discussion}

We find two conditions to observe jet impact, one of sufficient velocity and one of stability. 
The critical lines for the two conditions are shown on Figure 21. As $\beta$ decreases, the stability condition moves up. However, our numerics show that it does not intersect the sufficient velocity condition: near the critical $R e$, the jet always forms and what is relevant is the time it needs to reach the other side of the bubble. Thus in the sufficient velocity condition is the only relevant one. However, with surface tension added, it is possible that the jet formation condition could become relevant.

An interesting consequence of our theory is that it runs counter to a conventional point of view. It is generally considered that the more spherical the bubble, the later the jet develops and thus the stronger jet impact is. This is not consistent with our asymptotic scaling. In our theory $\beta$ controls bubble sphericity: the further the bubble is from the wall, the more spherical it remains at least initially. But at large $\beta$ the jet velocity is small

(Equation (5.14)). It is still true that for very early jet formation, 1) the momentum of the image bubble may not have enough time to be fully transmitted to the jet, and 2) impact may happen way before $R_{\min }$ which would reduce the jet velocity.

\section{Conclusions}

We have presented an original numerical technique to solve accurately the NavierStokes equations with free-surfaces. This method is not limited to simple geometries or small interface deformations. Particular emphasis has been put on the accurate description of free-surface boundary conditions. Validation tests have shown an excellent agreement with the Rayleigh-Plesset equation and a theoretical solution obtained by Prosperetti for the small amplitude shape oscillation of an ellipsoidal bubble.

Direct comparisons between high-speed photographic series and numerical simulations of bubble collapse near a solid boundary have shown a good qualitative and quantitative agreement while giving access to the details of the process. However, the simulations also show that viscous dissipation alone can not explain the strong damping of radial oscillations observed in the experiments. Acoustic and thermal dissipation - not taken into account in the present code - should be included in order to capture correctly the dynamics of the process after the first rebound. A simple solution would be to use a more sophisticated model equation for the pressure in the bubble.

A detailed parametric study of the influence of viscosity has demonstrated the existence of a critical value of the Reynolds number below which jet impact is no longer possible. A simple scaling law is shown to relate the value of this critical Reynolds number to two other non-dimensional parameters controlling the problem: the relative stand-off distance and the relative initial radius of the bubble.

We have presented a simple theory which describes correctly the overall characteristics of the phase diagram we obtain. In particular, we demonstrate the existence of a vertical asymptote in the parameter space of the rescaled non-dimensional control parameters. This provides a simple upper-bound for the domain in which jet impact is possible, independently of the Reynolds number.

A number of further studies would be possible and useful. In order to reduce the number of free parameters in the problem, we have neglected surface tension and chosen a constant polytropic exponent for the gas law. A simple analysis of the effect of surface tension is to compute a time scale related to surface tension near $R_{m}$. If this time scale is longer than $t_{m}$ then surface tension is negligible with respect to the only time scale appearing in the analysis of stability and jet velocity. The relevant dimensionless number 
is, going back to our first notations:

$$
N=\frac{\sigma_{\star} t_{m}^{2}}{R_{m}^{3}} .
$$

Using the above estimates, for $\gamma=7 / 5, N=\sigma_{\star} \alpha^{-1 / 4}=\left[\sigma /\left(R_{0} p_{\infty}\right)\right]\left(R_{M} / R_{0}\right)^{-1 / 4}$. It is interesting to apply this to the strong compression ratios in sonoluminescent air bubbles, which are very small. For a 5 micron air bubble, with $\alpha=10, N \sim 0.008$. For typical experiments on bubble collapse with larger $(1 \mathrm{~mm})$ bubbles, $N$ is even smaller. So for several practical applications neglecting surface tension is justified.

On the other hand, a parametric study of the influence of the polytropic exponent would allow to confirm the generality of the scaling laws we have found numerically and predicted theoretically. Moreover, while adequate for describing the general picture, the simple theory we propose is not able to explain the fact that the scaling we find numerically is clearly valid not only in the inviscid limit but across the whole range of Reynolds numbers we investigated. This remains an open question.

From a more practical point of view, it would be interesting to investigate how our phase diagram for jet impact influences our understanding of cavitation damage for real distributions of bubble sizes in experiments of hydrodynamic cavitation. If a majority of cavitation bubbles fall in the zone of the phase diagram where no jet impact is possible then cavitation damage would most probably be due only to the overpressure caused by the bubble collapse.

\section{REFERENCES}

Benjamin, T. B. \& Ellis, A. T. 1966 The collapse of cavitation bubbles and the pressure thereby produced against solid boundaries. Phil. Trans. Royal Soc. London A 260, 221240 .

Blake, J. R., Hooton, M. C., Robinson, P. B. \& Tong, R. P. 1997 Collapsing cavities, toroidal bubbles and jet impact. Phil. Trans. R. Soc. Lond. A 355 (1724), 537-550.

Blake, J. R., Robinson, P. B., Shima, A. \& Tomita, Y. 1993 Interaction of two cavitation bubbles with a rigid boundary. J. Fluid Mech. 255, 707-721.

Blake, J. R., Tomita, Y. \& Tong, R. P. 1998 The art, craft and science of modelling jet impact in a collapsing cavitation bubble. Appl. Sci. Res. 58, 77-90.

Blanco, A. \& Magnaudet, J. 1995 The structure of the axisymmetric high-reynolds number flow around an ellipsoidal bubble of fixed shape. Phys. Fluids 7 (6), 1265-1274.

Chan, R. K. C. \& Street, R. L. 1970 A computer study of finite-amplitude water waves. J. Comp. Phys. 6, 68-94.

Harlow, F. H. \& WELCh, J. E. 1965 Numerical calculation of time-dependent viscous incompressible flow of fluid with free surface. Phys. Fluids 8, 2182-2189.

HirT, C. W. \& Nichols, B. D. 1981 Volume of fluid VOF method for the dynamics of free boundaries. J. Comp. Phys. 39, 201-225.

Kornfeld, M. \& Suvorov, L. 1944 On the destructive action of cavitation. J. Appl. Phys. 15, 495-506.

Lamb, H. 1932 Hydrodynamics, 6th edn. Cambridge University Press.

LAUterborn, W. \& Bolle, H. 1975 Experimental investigations of cavitation-bubble collapse in the neighbourhood of a solid boundary. J. Fluid Mech. 72, 391-399.

Lauterborn, W. \& OhL, C. D. 1997 Cavitation bubble dynamics. Ultrasonics Sonochemistry $4,65-75$.

LEGENDRE, D. 1996 Quelques aspects des forces hydrodynamiques et des transferts de chaleur sur une bulle sphérique. PhD thesis, Institut National Polytechnique de Toulouse, France.

NAudÉ, C. F. \& Ellis, A. T. 1961 On the mechanism of cavitation damage by nonhemispherical cavities collapsing in contact with a solid boundary. Trans. ASME D: Basic Eng. 83, 648-656. 
Nichols, B. D. \& HiRT, C. W. 1971 Improved free surface boundary conditions for numerical incompressible-flow calculations. Journal of Computational Physics 8, 434-448.

Peyret, R. \& Taylor, T. D. 1983 Computational Methods for Fluid Flow. New York/Berlin: Springer Verlag.

Philipp, A. \& Lauterborn, W. 1998 Cavitation erosion by single laser-produced bubbles. J. Fluid Mech. 361, 75-116.

Plesset, M. S. \& Chapman, R. B. 1971 Collapse of an initially spherical vapour cavity in the neighbourhood of a solid boundary. J. Fluid. Mech. 47, 283-290.

Plesset, M. S. \& Prosperetti, A. 1977 Bubble dynamics and cavitation. Ann. Rev. Fluid Mech. 9, 145-185.

Popinet, S. \& ZALESKi, S. 1999 A front tracking algorithm for the accurate representation of surface tension. Int. J. Numer. Meth. Fluids 30, 775-793.

Press, W. H. \& Teukolsky, S. A. sep/oct 1991 Multigrid methods for boundary value problems. Computers in Physics pp. 514-519.

Prosperetti, A. 1980 Free oscillations of drops and bubbles: the initial-value problem. J. Fluid Mech. 100, 333-347.

Rattray, M. 1951 Perturbation effects in bubble dynamics. Technical report. California Institute of Technology.

RAYleigh, L. 1917 On the pressure developed in a liquid during the collapse of a spherical cavity. Phil. Mag. 34, 94-98.

TомiтA, Y. \& Shima, A. 1986 Mechanisms of impulsive pressure generation and damage pit formation by bubble collapse. J. Fluid. Mech. 169, 535-564. 